\title{
Preferences, Lévy Jumps and Option Pricing
}

\author{
Chenghu $\mathrm{Ma}^{1}$ \\ WISE, Xiamen University, China 361005 \\ E-mail: cma2006@xmu.edu.cn \\ Phone: 86-592-2181602
}

November 15, 2007

\begin{abstract}
${ }^{1}$ Some of the results reported in this paper were circulated through a working paper of mine entitled: "Valuation of Derivative Securities with Mixed Poisson-Brownian Information and Recursive Utility", 1993 McGill University Working Paper Series. I am grateful to Phelim Boyle, Darrell Duffie, Larry Epstein, Shige Peng, Ken Siu, Howard Thompson, Kenneth Vetzal, Tan Wang, Wing Keung Wong and referees for useful comments and discussions on previous drafts of the paper. Financial support from Xiamen University (China), ESRC research grant (UK) and SSHRC (Canada) are gratefully acknowledged. I assume responsibility for the content.
\end{abstract}




\begin{abstract}
This paper derives an equilibrium formula for pricing European options and other contingent claims which allows incorporating impacts of several important economic variable on security prices including, among others, representative agent preferences, future volatility and rare jump events. The derived formulae is general and flexible enough to include some important option pricing formulae in the literature, such as Black-Scholes, Naik-Lee, Cox-Ross and Merton option pricing formulae. The existence of jump risk as a potential explanation of the moneyness biases associated with the Black-Scholes model is explored.

Key Words: equilibrium option pricing, recursive utility, Levy jumps.

JEL Classification: G10, G11, G12, G13

Relevance to Practice: Research conducted in this paper identifies a unique role of financial options for revealing future volatility, magnitude and frequency of forthcoming rare jump event, along with representative agent's utility function. With the derived option pricing formula, one may readily applied the well developed technique of Laplace transform or fast Fourier transform to tackle inversion problem of this sort. These are of great potential value to practitioners, particularly for managing risk in a period of financial crises experienced frequently in recent history.
\end{abstract}




\section{Introduction}

This paper derives an option pricing formula in a continuous time jump diffusion framework. It generalizes the formula derived by Naik and Lee (1990) in two respects: First, it permits the magnitude of the jump to follow any distribution with finite moments, and second, the utility function is recursive but is not necessarily an intertemporal additive von-Neumann Morgenstern utility function. The usefulness of the recursive utility specification is reflected not only from its mathematical generality and flexibility offered, but also from the predictions and economic implications generated by non-expected utility functions that are in line with agent's choice behaviour in presence of risk. The latter refers to its ability to resolve several documented paradoxical behaviour in contexts of choice under risk. These include the well-known Allais paradox (see Epstein 1992).

Several papers have addressed the issue on the existence of jumps and their effects on the dynamics of security prices and, in particular, on derivative securities. Press (1967) is probably among the first to point out that a diffusion price process with jumps could be one way to model security price movements. Substantial empirical findings reported by Brown and Dybvig (1986) and Jarrow and Rosenfeld (1984) confirm Press's observation. Cox and Ross (1976), Merton (1976) and Naik and Lee (1990) each introduce Poisson jumps into option pricing models, and all lead to the conclusion that the existence of jumps could be one of the crucial factors in determining the prices of the options. Finally, as to be explained in section 4 of this paper, introducing the jump risk could be potentially useful to explain the empirical biases associated with the Black-Scholes model, which thus also help to resolve the volatility smile puzzle originated from Bailey and Stulz (1989).

Given its empirical advantage in modelling security price movements, introducing the Lévy jump process into the theoretical framework also creates some serious challenges for economists and market practitioners. As pointed out by Naik and Lee (1990), it is difficult to price derivative securities in the presence of Lévy jumps because the capital market is generically incomplete. In other words, the payoffs of the derivative securities may not be replicated perfectly by portfolios of primitive securities. As a consequence, the arbitrage free approach for pricing derivative securities as applied by Black and Scholes (1973) in a pure Brownian information world does not apply to the Lévy jump framework; that is, the no-arbitrage condition alone is not enough to determine a unique price for a derivative security. ${ }^{1}$

Different approaches have been proposed in literature for pricing derivative securities in an incomplete market. These include the approach of (quadratic)

\footnotetext{
${ }^{1}$ The case studied by Cox and Ross (1976), which assumes pure Poisson process with constant jump size, is actually identified as the unique situation where the capital market becomes complete with the market portfolio and risk free bonds as tradable securities. This is in addition to the case of no jumps as assumed by Black and Scholes (1973). Merton (1976) derived an option pricing formula by assuming zero correlation between returns of the derivative securities and that from the market portfolio.
} 
cost minimization for hedging a contingent claim formulated by Föllmer and Sandermann (1986) and Föllmer and Schweizer (1991), the Esscher transformation approach proposed by Gerber and Shiu (1994), and Naik and Lee (1990)'s equilibrium option pricing under the assumption of representative agent of expected additive utility. See also Davis (1997) who considers utility maximization for option pricing.

Following Naik and Lee (1990), this paper considers the equilibrium option pricing problem in a continuous time analog of the Lucas (1978) exchange economy under the assumption of a representative agent. The agent maximizes a so-called intertemporal stochastic differential utility of Duffie and Epstein (1992 a) and Ma (2000). The stochastic differential utility is regarded as a continuous time analog of Epstein and Zin (1989)'s recursive utility formulated in discrete time. This paper contains, as promised, a derivation of a general option pricing formula when asset return follows a geometric Lévy process in extending the geometric Brownian motion specification assumed in Black and Scholes (1973).

One merit of the derived option pricing formula involves its advantages in addressing the issues related to the empirical biases associated with the BlackScholes option pricing formula, and concerning the behavioral assumption with respect to representative agent's utility function. In an accompanying paper, Ma and Vetzal (1995) conducted several numerical experiments to examine how changes in preference parameters and jump size distributions, in addition to drift and volatility that govern the motion of stock prices, etc, may affect the option prices.

The derived option pricing formula is also useful for examining how the representative agent's preference specifications may affect the equilibrium security prices, and conversely how the equilibrium security prices reveal information about the representative agent's utility function. For example, one may ask: is it possible to distinguish between the class of non-expected recursive utility functions and the traditional expected additive utility functions from the underlying equilibrium security prices? In a separate paper, Ma (2005) proved that the option pricing formula corresponding to the stochastic differential utilities of Duffie and Epstein (1992 a) and Ma (2000) are observational distinguishable from the ones generated by specifying the von Neumann and Morgenstern (1947) and Kreps and Porteus (1979) expected utility functions. Similar exercises are carried out by Ma (1998) in a discrete time framework.

Mathematically, the relevant option pricing formula admits a simple expression. It is expressed in terms of the Laplace inverse transformation of a complex-valued function $\Phi(\cdot)$, the so-called transmitting function. Preference parameters and other aggregate economic factors, namely, the future volatility, the frequency and the magnitude of shifts in asset price in event of rare jumps, affecting the option prices are explicitly incorporated through the transmitting function. It also yields a general closed form formula for European call options since it nests many existing models in the literature. For example, the option pricing formulae derived by Black-Scholes, Naik-Lee, Cox-Ross and Merton can all be represented in terms of Laplace inverse transforms of some suitably defined $\Phi(\cdot)$-functions. 
The rest of the paper is organized as follows. The primitives of the model, including the information structure, market structure and agents' preferences are described in section 2. In section 3, the representative agent's optimal choice problem is formulated together with a derivation of an equilibrium asset pricing formula. The latter is achieved by introducing the so-called pseudostate process. Section 4 considers a parametric specification of the economy studied in the previous section. This section derives a closed-form formula for European call options as well as for other derivative securities. It also provides a comparison of the derived option pricing formula with existing formulae in the literature, together with a discussion on moneyness biases associated with the Black-Scholes model as a consequence of ignoring the jump risk in security price movements. Section 5 contains some concluding remarks. The appendix contains some of the proofs.

\section{The Primitives}

This section contains a description of the primitives of an economy including the information structure, the market structure and the agent's utility function.

\subsection{The Information Structure}

We assume as given a complete filtered probability space $\{\Omega, \mathbb{F}, P\}$ with an increasing and right continuous complete filtration $F=\left\{\mathbb{F}_{t}\right\}_{t \geqslant 0}$. The probability space describes the uncertainty of the economy, while the information filtration models the flow of information. The right continuity property of the information filtration is to ensure that there is no surprise to the flow of information at any specific point of time. The increasing property of the information filtration is also known as "the long memory" assumption. It suggests that all recorded past events remain to be recorded in the current information set.

Let $N=\left\{N_{t}\right\}_{t \geq 0}$ be an $\mathbb{F}$-adapted Lévy process, ${ }^{2}$ and let $\Delta N_{t} \equiv N_{t}-N_{t-}$ denote the size of a jump that occurs to the jump process at time $t$. For any $\Gamma \in \mathcal{B}(\mathbb{R}), v(t, \Gamma) \equiv \sum_{0<s<t} 1_{\Gamma}\left(\Delta N_{s}\right)$ defines a $\mathbb{F}$-adapted Poisson process $\{v(t, \Gamma)\}_{t>0}$ with parameter $\nu(\Gamma)=\mathbb{E}[v(1, \Gamma)] \geq 0$, where $1_{\Gamma}(u)=1$ and 0 respectively for $u \in \Gamma$ and $u \notin \Gamma$. $v(t, \Gamma)$ counts the number of jumps of the process $N$ with sizes in $\Gamma$ in the time interval $[0, t]$. The measures $v(\cdot, \cdot)$ and $\nu(\cdot)$ are respectively referred to as the Poisson random measure and the Lévy measure for the Lévy process $N$.

By definition, the Lévy measure $\nu(\cdot): \mathcal{B}(\mathbb{R}) /\{0\} \rightarrow \mathbb{R}_{+}$specifies the statistical properties of the Poisson random measure. It can be regarded as a measure of relative frequency of the jumps within different size categories. For example,

\footnotetext{
${ }^{2}$ A real valued process $\left\{x_{t}\right\}_{t \geq 0}$ is called $\mathbb{F}$-adapted if $x(t, \cdot)$ is $\mathbb{F}_{t}$-measurable for each $t \geq 0$; that is, $x_{t}$ is known given $\mathbb{F}_{t} .\left\{x_{t}\right\}_{t>0}$ is called $\mathbb{F}$-progressively measurable if $x(\cdot, \cdot):[0, t] \times \Omega$ $\rightarrow \mathbb{R}$ is $\mathcal{B}([0, t]) \times \mathbb{F}_{t}$-measurable for each $t \geq 0$, where $\mathcal{B}([0, t])$ is the Borel $\sigma$-algebra on $[0, t]$. Finally, a Lévy process is an $\mathbb{F}$-adapted process that has stationary and independent increments, and is continuous in probability (see Protter 1990).
} 
for $\Gamma_{1}, \Gamma_{2} \in \mathcal{B}(\mathbb{R}) /\{0\}, \Gamma_{1} \cap \Gamma_{2}=\emptyset$, the inequality $\nu\left(\Gamma_{1}\right)<\nu\left(\Gamma_{2}\right)$ can be interpreted as follows: for any given time period, more jumps with sizes in $\Gamma_{2}$ than those in $\Gamma_{1}$ will be expected.

There are two independent sources of uncertainty in this model. The first is from an $m$-dimensional standard Brownian motion $\left\{B_{t}\right\}_{t>0}$ on $\{\Omega, \mathbb{F}, P\}$ with respect to its own information filtration $\{\Omega, \mathbb{F}, P\}$. The Brownian motion has continuous sample paths. The other source of uncertainty comes from the Lévy jump process $\left\{N_{t}\right\}_{t>0}$ described above. These two sources of uncertainty affect the state of nature through the state process $\left\{x_{t}\right\}_{t>0}$, which is $\mathbb{R}^{m}$-valued, $\mathbb{F}$ progressively measurable on $\mathbb{R}_{+} \times \Omega$. The motion of the state process $\left\{x_{t}\right\}_{t \geq 0}$ is assumed to follow a stochastic differential-difference equation (SDDE):

$$
d x_{t}=b\left(t, x_{t-}\right) d t+a\left(t, x_{t-}\right) d B_{t}+\int_{\mathbb{R}} l\left(t, x_{t-}, u\right) v(d t, d u),
$$

where $x_{0}$ is the given initial state, and $b: \mathbb{R}_{+} \times \mathbb{R}^{m} \rightarrow \mathbb{R}^{m}, a: \mathbb{R}_{+} \times \mathbb{R}^{m} \rightarrow \mathbb{R}^{m \times m}$ and $l: \mathbb{R}_{+} \times \mathbb{R}^{m} \times \mathbb{R} \rightarrow \mathbb{R}^{m}$ are non-random continuous functions of dimension $m \times 1, m \times m$ and $m \times 1$ respectively. This notion of SDDE is taken from Gihman and Skorohod (1972). ${ }^{3}$ The coefficients in (1) are assumed to satisfy the Lipschitz and growth conditions to ensure equation (1) admits a unique right continuous solution (See Gihman and Skorohod 1972, §3 Theorem 2 and $\S 7$ Theorem 1, Part II).

In equation (1), when a jump of size $u$ occurs at time $t$, i.e., $\triangle N_{t}=u$, the corresponding state variable jumps from $x_{t-}$ to $x_{t+}=x_{t-}+l\left(t, x_{t-}, u\right)$. The subscripts " \pm " are to emphasize that a jump occurs at time $t$, which is used throughout this paper for all random variables that are functions of the state variables. Sometimes we drop the subscripts for notational simplicity without causing confusion.

\subsection{The Market Structure}

Consider a continuous-time version of the Lucas (1978) exchange economy with information structure and state space described above. Assume that there is one consumption good and $n+1$ assets traded in the market at each point of time. Asset 0 is a risk-free bond with time $t$ interest rate $r\left(t, x_{t}\right)$, and assets 1 through $n$ are risky with non-negative dividend rates $\delta^{j}\left(t, x_{t}\right)$ respectively for $j=1, \ldots, n$, all affected by the realization of the state variables $x_{t}$. The interest rate $r$ and dividend rates $\delta$ are expressed in units of the consumption good. Assume further that the asset price processes $S_{t}=\left[S_{t}^{1}, \cdots, S_{t}^{n}\right]^{\top}$ satisfy SDDE

$$
\begin{aligned}
d S_{t}= & {\left[\lambda\left(t, x_{t-}\right) \circ S_{t-}+r\left(t, x_{t-}\right) S_{t-}-\delta\left(t, x_{t-}\right)\right] d t+S_{t-} \circ \sigma\left(t, x_{t-}\right) d B_{t} } \\
& +S_{t-} \circ \int_{\mathbb{R}} \gamma\left(t, x_{t-}, u\right) v(d t, d u)
\end{aligned}
$$

\footnotetext{
${ }^{3}$ Protter (1990) considers SDDE for general semi-martingales that contain the Lévy process described here as a special case. The corresponding SDDE for Lévy processes admit the same mathematical expression as equation (1) following the Lévy Decomposition Theorem (Protter 1990, Theorem 42 in Chapter 1), and from the definition of stochastic integration (Protter 1990, pp.50-51).
} 
with $S_{0}$ given. ${ }^{4}$ The coefficients in equation (2) are such that

$$
\begin{aligned}
\lambda(t, x) & =\left[\lambda^{1}(t, x), \cdots, \lambda^{n}(t, x)\right]^{\top} \\
\delta(t, x) & =\left[\delta^{1}(t, x), \cdots, \delta^{n}(t, x)\right]^{\top} \\
\sigma(t, x) & =\left[\sigma^{1}(t, x), \cdots, \sigma^{n}(t, x)\right]^{\top} \\
\gamma(t, x, u) & =\left[\gamma^{1}(t, x, u), \cdots, \gamma^{n}(t, x, u)\right]^{\top}
\end{aligned}
$$

where $\lambda^{j}, \delta^{j}$ and $\gamma^{j}$ are respectively the drift, the dividend rate and the size of the jump for the underlying security $j$, and where $\sigma^{j}$ is a $m$-dimensional column vector that determines the coefficients of the security $j$ with respect to the $m$ dimensional Brownian motion $\left\{B_{t}\right\}_{t \geq 0}$. It is noted that, the instantaneous unit risk premium for security $j$ is thus given by $\lambda^{j}(t, x)+\int_{\mathbb{R}} \gamma^{j}(t, x, u) \nu(d u)$, and that the instantaneous volatility can be decomposed into $\sigma^{j}(t, x)^{\top} \sigma^{j}(t, x)+$ $\int_{\mathbb{R}}\left[\gamma^{j}(t, x, u)\right]^{2} \nu(d u)$.

We assume that $r(\cdot, \cdot), \lambda(\cdot, \cdot), \sigma(\cdot, \cdot)$ and $\int_{\mathbb{R}} \gamma(\cdot, \cdot, u) v(d t, d u)$ defined on $(t, x) \in \mathbb{R}_{+} \times \mathbb{R}^{m}$ are continuously differentiable and uniformly bounded, and have bounded first order derivatives in both arguments. The dividend rate $\delta(\cdot, \cdot)$ defined on $\mathbb{R}_{+} \times \mathbb{R}^{m}$ is assumed to be continuously differentiable with bounded first order derivatives in both arguments. These assumptions will ensure the asset price process as a unique solution to the SDDE (2) to be well defined.

In addition, we assume that the dividend rates $\delta^{j}, j=1, \cdots, n$, fall into the positive cone of a so-called consumption space $\mathbb{D}$. The consumption space $\mathbb{D}$ is assumed to be a complete metric space/Banach space that contains $\mathbb{R}_{+}$-valued and $\mathbb{F}$-adapted right continuous processes $d \equiv\left\{c_{t}\right\}_{t>0}$. One may imagine the consumption space to contain all possible cash flows that are achievable by trading among these above specified $n+1$ tradable securities and by restricting the portfolio holdings to be within a so-called admissible set $\Lambda$. Precise formulation of admissible cash flows will be given in Section 3.1.

\subsection{The Preference System}

Given the filtered probability space $\{\Omega, \mathbb{F}, P\}$ with $\mathbb{F}=\left\{\mathbb{F}_{t}\right\}_{t \geqslant 0}$ as described above, and given a consumption space $\mathbb{D}$, agent's preference over consumption streams in $\mathbb{D}$ is thus summarized by a so-called preference system $\succsim=$ $\left\{\succsim_{\left(t, \mathbb{F}_{t}\right)}\right\}_{t \geq 0}$ such that, for all $t, \succsim_{\left(t, \mathbb{F}_{t}\right)} \subseteq \mathbb{D} \times \mathbb{D}$ is a preference relation that represents agent's time- $t$ preference over all consumption programs in $\mathbb{D}$, taking as given the time-t information $\mathbb{F}_{t}$. In many economic applications, the preference system is assumed to satisfy certain desirable properties such as monotonicity, continuity, and dynamic consistency. ${ }^{5}$ Under these conditions, the preference

\footnotetext{
${ }^{4}$ In equation (2), for all matrix $A, A^{\top}$ is the transpose of $A$. The operator $\circ: \mathbb{R}^{n} \times \mathbb{R}^{n} \rightarrow \mathbb{R}^{n}$ is a product operation defined on the Euclidean space $\mathbb{R}^{n}$ such that $v \circ w=\left[v_{1} w_{1}, \cdots, v_{n} w_{n}\right]$ for all $v$ and $w$ in $\mathbb{R}^{n}$. The inner product between $v$ and $w$ is denoted as $v \cdot w=\sum_{i=1}^{n} v_{i} w_{i}$.

${ }^{5}$ For example, we say that $\succsim$ displays dynamically consistency if, for all $d$ and $d^{\prime}$ such that $d \succ_{\left(\tau, \mathbb{F}_{\tau}\right)} d^{\prime}$ and that $c_{t}=c_{t}^{\prime}$ for all $t \leq \tau$, then $d \succ_{\left(t, \mathbb{F}_{t}\right)} d^{\prime}, \forall t \leq \tau$.
} 
can be represented by some well-defined utility functions $V_{t}: \mathbb{D} \longrightarrow \mathbb{R}$ such that

$$
d \succ_{\left(t, \mathbb{F}_{t}\right)} d^{\prime} \Leftrightarrow V_{t}\left(d \mid \mathbb{F}_{t}\right)>V_{t}\left(d^{\prime} \mid \mathbb{F}_{t}\right), t \geq 0 .
$$

For example, the von Neumann and Morgenstern (1947) expected additive utility in continuous time admits the following representation: For all $d=\left\{c_{t}\right\}_{t \geq 0} \in$ $\mathbb{D}$,

$$
V_{t}\left(d \mid \mathbb{F}_{t}\right)=\mathbb{E}_{t}\left[\int_{t}^{\infty} e^{-\beta(s-t)} u\left(c_{s}\right) d s\right], t \geq 0
$$

where $\beta>0$ is a time preference parameter that measures investor's impatience over future cash flows, and $u: \mathbb{R}_{+} \rightarrow \mathbb{R}$ is a utility index, and where $\mathbb{E}_{t}[\cdot]$ stands for the conditional expectation given the time- $t$ information $\mathbb{F}_{t}$.

In the rest of the paper, we consider a continuous time analog of a discretetime intertemporal recursive utility originated from Epstein and Zin (1989). ${ }^{6}$ It is first formulated by Duffie and Epstein (1992 a), and is further extended by Ma $(1992,2000)$ to accommodate the mixed Poisson-Brownian information structure.

An utility function is defined by two primitive functions $f(\cdot, \cdot)$ and $M(\cdot, \cdot)$, which are assumed to be continuously differentiable in addition to some other regularity conditions (see Ma 2000). The pair $(f, M)$ is known as utility generator. Moreover, $f$ determines the 'certainty preference', while suitable changes in $M$, with $f$ fixed, correspond to changes in the degree of risk aversion. For a given consumption program $\left\{c_{t}\right\}_{t>0}$ in the consumption space $\mathbb{D}_{+}$, the utility process $\left\{V_{t}\right\}_{t>0}$ is a unique integrable semi-martingale that solves the following backward stochastic integral equation

$$
V_{t}=\mathbb{E}_{t}\left[\int_{t}^{T}\left\{f\left(c_{s-}, V_{s-}\right)+\int_{\mathbb{R}} M\left(V_{s+}, V_{s-}\right) \nu(d u)\right\} d s+V_{T}\right]
$$

for all $0 \leq t \leq T<\infty$. In particular, we restrict $\left\{V_{t}\right\}_{t \geq 0}$ to satisfy an asymptotic boundary condition:

$$
\lim _{T \rightarrow \infty} \mathbb{E}_{t}\left[e^{\varphi T} V_{T}\right]=0, \forall t \geq 0
$$

for some positive constant $\varphi$. The asymptotic condition is to ensure the contribution (towards current utility) that is derived from utilities entertainable far

\footnotetext{
${ }^{6}$ For any given consumption program $\left\{c_{t} ; t=0,1, \ldots\right\}$, Epstein and Zin (1989) define the corresponding utility process recursively by

$$
V_{t}=\mathcal{W}\left(c_{t}, \mu\left(V_{t+1} \mid \mathbb{F}_{t}\right)\right),
$$

where $\mathcal{W}: \mathbb{R} \times \mathbb{R} \rightarrow \mathbb{R}$ is a utility aggregator, $\mu$ is a certainty equivalent operator that provides a rank of future random utility $V_{t+1}$ given the information $\mathbb{F}_{t}$. The conventional expected additive utility

$$
V_{t}=\mathbb{E}_{t}\left[\sum_{s=0}^{\infty} \beta^{s} u\left(c_{t+s}\right)\right], 0<\beta<1,
$$

is achieved by setting $\mathcal{W}(c, v)=u(c)+\beta v$, and the future random utility function is ranked by its conditional expectation given the information $F_{t}$ (i.e., $\mu\left(V_{t+1} \mid \mathbb{F}_{t}\right)=$ $\left.E_{t}\left[V_{t+1}\right]\right)$. See Chew and Epstein (1990) for an axiomatic foundation for recursive utility.
} 
into a future time $T$ is to decline at an exponential rate $\varphi$. This condition is naturally satisfied for all finite-lived agents.

Equation (5) is known as Koopmans equation à la Koopmans (1960). The existence of utility process as a unique solution to the Koopmans equation is studied by Duffie and Epstein (1992 a) and Ma (2000). For the following, we assume that

(U) The utility function $V_{t}: \mathbb{D}_{+} \rightarrow \mathbb{R}, t \geq 0$, as unique solution to the Koopmans equation (5), is well defined; namely, it is continuous, strictly monotonic increasing and strictly concave. Moreover, it is assumed to be Gateaux differentiable. ${ }^{7}$

There follows some typical examples of recursive utility:

Example 1 (Expected Additive Utility) The expected additive utility $\left\{V_{t}\right\}_{t>0}$ defined by equation (4) solves uniquely the Koopmans equation with utility generator $f(c, v)=u(c)-\beta v$ and $M(x, y)=0$.Here, $\beta>0$ is a time-preference parameter, while the utility index $u$ characterizes investor's attitudes towards risk.

Example 2 (Kreps-Porteus Utility) The KP expected utility generator in continuous time takes the form:

$$
f(c, v)=\frac{h^{\prime}\left(h^{-1}(v)\right)}{u^{\prime}\left(h^{-1}(v)\right)}\left[u(c)-\beta u\left(h^{-1}(v)\right)\right] \text { and } M(x, y)=0 .
$$

For deterministic consumption programs, the time-zero utility $V_{0}$ is given by

$$
V_{0}=h\left(u^{-1}\left[\int_{t \geq 0} e^{-\beta t} u\left(c_{t}\right) d t\right]\right)
$$

which is ordinal equivalent to the discounted utility $\int_{t>0} e^{-\beta t} u\left(c_{t}\right) d t$. Thus, the certainty preference, including intertemporal substitution and time preference, is determined by $u(\cdot)$ and $\beta$. A particular parameterization of the KP utility generator in discrete time is studied by Epstein and Zin (1989), and is achieved by specifying $u(c)=\frac{c^{\zeta}}{\zeta}$ and $h(x)=\frac{x^{\alpha}}{\alpha}$. Here, $1-\alpha$ measures the degree of risk aversion, and $(1-\zeta)^{-1}$ gives the degree of intertemporal substitution.

Example 3 (Betweenness Utility) The continuous-time betweenness utility generator $(f, M)$ is given by

$$
f(c, v)=\frac{u(c)-\beta u(\varphi(v))}{u^{\prime}(\varphi(v)) \varphi^{\prime}(v)} \text { and } M(x, y)=\frac{H(\varphi(x), \varphi(y))}{H_{1}(\varphi(y), \varphi(y)) \varphi^{\prime}(y)}-x+y
$$

\footnotetext{
${ }^{7}$ The utility gradient $\partial V(c, d)$ is defined as the Gateaux derivative of $V(c)$ in the direction $d$; i.e.,$$
\partial V(c, d) \equiv \lim _{\epsilon \rightarrow 0+} \frac{V(c+\varepsilon d)-V(c)}{\varepsilon}
$$

provided the limit takes a finite value. 
where $\varphi$ is a monotonic function with its inverse given by

$$
\varphi^{-1}(v)=\int_{v_{0}}^{v} \exp \left[\int_{v_{0}}^{u} \frac{H_{11}(x, x)}{H_{1}(x, x)} d x\right] d u .
$$

Here, $v_{0}$ is arbitrarily given, and $H$ is assumed to be twice continuously differentiable. Its first- and second-order partial derivatives with respect to the first argument that are evaluated at $(x, x)$ are respectively assumed to be positive and negative (i.e., $H_{1}(x, x)>0$ and $H_{11}(x, x)<0$ ). As in the previous example, $u$ and $\beta$ determine the certainty preferences, and $H$ provides an avenue through which risk aversion can be changed without affecting the certainty preference. ${ }^{8}$ For the special case of $H(x, y)=h(x)-h(y)$, equation (8) reduces to equation (7), the KP utility generator, with $\varphi(x)=h^{-1}(x)$.

\section{An Equilibrium Asset Pricing Model}

Following Lucas (1978), Merton (1973), and many others, in this section we formulate agent's consumption and portfolio choice problem as an optimal control problem. An equilibrium asset pricing model is derived following the utility gradient approach à la Duffie and Skiadas (1994).

\subsection{The Optimal Choice Problem}

Agent is endowed with an initial positive wealth, and is to choose a consumption and portfolio policy $\left\{\left(c_{t}, z_{t}\right)\right\}_{t>0}$ to maximize its life time utility. The policy, of course, must be budget feasible. As mentioned in the previous section, the consumption process is required to be in the positive cone of the consumption space $\mathbb{D}$. The portfolio process $\left\{z_{t}\right\}_{t \geq 0} \in \mathbb{Z}$ is restricted to be in the positive unit simplex

$$
\Lambda=\left\{z \in[0,1]^{n}: \sum_{i=1}^{n} z_{i} \leq 1\right\}
$$

and to be $\mathbb{F}$-progressively measurable. For all $j, z_{t}^{j}$ is the proportion of the wealth that is invested in security $j$ over all invested in risky securities at time $t$. Here we impose the no short-selling constraints which are necessary for the existence of a solution for agent's optimal choice problem described below.

Following a standard procedure developed by Merton (1973), for any given consumption and portfolio policy, the agent's flow budget constraint is shown

\footnotetext{
${ }^{8}$ Given two risk averse certainty equivalents $\mu_{1}$ and $\mu_{2}, \mu_{1}$ is said to be more risk averse than $\mu_{2}$ if, for all random variable $x$, we have: $\mu_{1}(x) \leq \mu_{2}(x) \leq \mathbb{E}[x]$. For betweenness utility generators $H$ and $H^{*}$, the condition under which the certainty equivalent associated with $H$ is to be more risk averse than that with $H^{*}$ is

$$
-\frac{H_{11}(x, x)}{H_{1}(x, x)} \leq-\frac{H_{11}^{*}(x, x)}{H_{1}^{*}(x, x)}, \forall x .
$$
}


to satisfy a stochastic differential and difference equation:

$$
\begin{aligned}
d W_{t}= & \left(W_{t-}\left[\lambda_{t-} \cdot z_{t-}+r_{t-}\right]-c_{t-}\right) d t+W_{t-} z_{t-} \cdot \sigma_{t-} d B_{t} \\
& +W_{t-} \int_{\mathbb{R}} z_{t-} \cdot \gamma_{t-} v(d t, d u)
\end{aligned}
$$

where $W_{t}$ is the time- $t$ wealth with initial wealth $W_{0}>0$ as given. For notational simplicity, we use the subscript $t$ to denote the underlying random variables.

A policy $(c, z) \in \mathbb{D} \times \mathbb{Z}$ is called admissible if equation (9), together with (1) and (2), admits a unique non-negative solution $W$. In that case, we say that the consumption program $c$ is financed by the portfolio policy $z$. Given the initial state $\left(x_{0}, W_{0}\right)$ at $t=0$, we denote by $\mathcal{C}_{0} \subset \mathbb{D} \times \mathbb{Z}$ the set of all admissible policies. The set $\mathcal{C}_{0}$ is obviously not empty.

A feasible policy $(c, z) \in \mathcal{C}_{0}$ is optimal if it achieves the value of the following optimization problem:

$$
U\left(0, x_{0}, W_{0}\right)=\sup _{\substack{(c, z) \in \mathcal{C}_{0} \\ \text { s.t. }(5) \&(6)}} V_{0} .
$$

Or, in words, an optimal policy is an admissible policy that maximizes agent's utility function among all admissible consumption programs.

Remark 4 The above formulated optimal choice problem as the optimal control problem is studied by Ma (1992, 2001). The Bellman equation, to be sufficient condition for the optimal choice problem, is derived there together with a derivation of Euler equations.

\subsection{Equilibrium}

Assume that the representative agent is endowed with one share of each of the risky securities, and that the aggregate dividend rate process $\delta^{m} \in \mathbb{D}$ is strictly positive almost everywhere.

A competitive equilibrium of the economy is a set of security price processes such that, given the budget and the other feasibility constraints, the representative agent optimally chooses to hold one share of each of the securities and to consume the aggregate dividend; i.e.,

$$
z^{j}=\frac{S^{j}}{\sum_{j=1}^{n} S^{j}} \text { and } c=\sum_{j=1}^{n} \delta^{j}=\delta^{m}
$$

constitutes an optimal policy with wealth $W=\sum_{j=1}^{n} S^{j}=S^{m}$ coinciding with the price of the market portfolio. Here, $m$ stands for the market portfolio, or the aggregate equity.

Lemma 5 If the equilibrium exists, then the underlying equilibrium price processes must solve the following stochastic integral equations

$$
S_{t}^{j}=\frac{1}{\pi_{t}} \mathbb{E}_{t}\left[\int_{t}^{T}\left\{\pi_{s_{-}} \delta_{s-}^{j}+\int_{\mathbb{R}} M_{1+} \pi_{s+} S_{s+}^{j} v(d u)\right\} d s+\pi_{T} S_{T}^{j}\right]
$$


for all $T \geq t \geq 0, j=1, \ldots, n$, where

$$
\pi=e^{\int_{0}^{t} \rho_{s} d s} f_{c}\left(\delta^{m}, V\right) \text { with } \rho=f_{v}\left(\delta^{m}, V\right)+\int_{\mathbb{R}} M_{2+} v(d u)
$$

where $M_{i+}$ stands for $M_{i}\left(V_{+}, V\right), i=1,2, f_{c}$ and $f_{v}$ are respectively the partial derivatives of $f$ with respect to its first-and second arguments, and $V=V\left(\delta^{m}\right)$.

Proof. See Ma (2006, Proposition 10).

Remark 6 Equation (11) corresponds to the Euler equation for representative agent's optimal choices when optimal consumption is set at the aggregate dividend. For the special cases of no jumps, the equation (11) reduces to the standard Arrow-Debreu security price formulation. In these cases, $\pi$ is referred to as the "state price process", which is common to all securities. In the presence of Lévy jumps with non-expected utility, the current price defined by the Euler equation is affected not only by its future dividend streams but also by its future price movements. Accordingly, $\pi$ is referred to as the "pseudo-state price process".

To solve the equation (11) for the equilibrium prices, we need to introduce a so-called pseudo state variables:

Let $\widehat{v}(\cdot, \cdot)$ be a Poisson random measure with its Lévy measure $\widehat{\nu}(\cdot)$ to be given by

$$
\widehat{\nu}_{s}(d u)=\frac{\left(M_{1}\left(V_{s+}, V_{s-}\right)+1\right) f_{c}\left(c_{s+}, V_{s+}\right)}{f_{c}\left(c_{s-}, V_{s-}\right)} \nu(d u)
$$

in which $c=\left\{\delta^{m}\left(t, x_{t}\right)\right\}_{t>0}$ and $V=\left\{V_{t}(c)\right\}_{t>0}$ respectively stand for the equilibrium aggregate consumption and the corresponding utility. The newly introduced Poisson random measure is, in general, time-varying and state-dependent.

The pseudo state process, denoted by $\widehat{x}$, is defined as the unique semimartingale that solves the following SDDE

$$
d \widehat{x}_{t}=\widehat{b}\left(t, \widehat{x}_{t-}\right) d t+a\left(t, \widehat{x}_{t-}\right) d \widehat{B}_{t}+\int_{\mathbb{R}} l\left(t, \widehat{x}_{t-}, u\right) \widehat{v}(d t, d u)
$$

with $\widehat{x}_{0}=x$, where $\widehat{b}=b+a a^{\top}\left(\frac{f_{c c}}{f_{c}} c_{x}+\frac{f_{c v}}{f_{c}} V_{x}\right), f_{c c}$ and $f_{c v}$ correspond to the second order partial derivatives for $f$ to be evaluated at $(c, V)$, and $\widehat{B}=\left\{\widehat{B}_{t}\right\}$ is a $m$-dimensional Brownian motion that is independent to the Lévy jumps summarized by $\widehat{v}(\cdot, \cdot)$.

We have:

Proposition 7 If the equilibrium exists, then the underlying equilibrium price process must be such that

$$
\begin{aligned}
S^{j}\left(t, x_{t}\right)= & \mathbb{E}_{t}\left[\int_{t}^{T} e^{-\int_{t}^{s} r\left(\tau, \widehat{x}_{\tau-}\right) d \tau} \delta^{j}\left(s, \widehat{x}_{s-}\right) d s\right] \\
& +\mathbb{E}_{t}\left[e^{-\int_{t}^{T} r\left(\tau, \widehat{x}_{\tau-}\right) d \tau} S^{j}\left(T, \widehat{x}_{T}\right)\right] \\
\forall T \geq & t \geq 0, j=1, \ldots, n
\end{aligned}
$$


in which

$$
r\left(t, x_{t}\right)=-\left\{f_{v}+\int_{\mathbb{R}} M_{2+} \nu(d u)+\frac{\mathcal{D} f_{c}}{f_{c}}\right\}+\nu(\mathbb{R})-\widehat{\nu}_{t}(\mathbb{R})
$$

is the equilibrium interest rates $r\left(t, x_{t}\right)$, where $D$ is the diffusion operator induced by the diffusion component of the jump-diffussion state process $\left\{x_{t}\right\}$; that is, for all $h \in C^{(1,2)}\left(\mathbb{R}_{+} \times \mathbb{R}^{m}\right)$, the diffusion operator acting on $h$ is defined by setting

$$
\mathcal{D} h(t, x) \equiv \frac{\partial h}{\partial t}+\frac{\partial h}{\partial x} \cdot b+\frac{1}{2} \operatorname{tr}\left(\frac{\partial^{2} h}{\partial x^{2}} a a^{\top}\right) .
$$

Proof. See Appendix A.

According to pricing formula (15), price equals to the present value of future cash flows with discount rates to be given by the risk free interest rates. In contrast to the traditional risk-neutral pricing rule of no-arbitrage, the expectation is not computed with respect to the original state process, but instead it is computed for the risk-adjusted and preference-dependent pseudo-state process. The change of the state processes plays essentially the same role as the change of probability measures in the no-arbitrage condition (Re: Harrison and Kreps 1979). The precise mathematical connection between change of probability measures and change of state processes (into pseudo-process) can be established. We choose not to cover this here because its coverage does not enhance much our understanding to the theory developed below in this paper. The mathematical convenience resulting from the use of the pseudo-state process, relative to the use of the risk-neutral measure, for our analysis is self-evident. The latter involves the use of the so-called "generalized Girsanov theorem" for changing of measures in presence of Lévy jumps, the proof of which itself represents a new mathematical result and is less known in economic literature. To interested readers, please refer to Ma (2002) for a proof of the generalized Girsanov theorem for jump-diffusion process.

Remark 8 The interest rate process $\left\{r\left(t, x_{t}\right)\right\}$ given by equation (16) has a natural interpretation as the dividend rate at which a security has a constant unit price. This formulation of short term interest rate process along with the corresponding term structure includes that of Duffie and Epstein (1992 b) as well as that of Cox, Ingersoll and Ross (1985).

Remark 9 The derived asset pricing model holds true at all terminal dates $T$, which could be a random stopping time or be deterministic. It also covers the case when the underlying security has a pre-specified lump-sum payoff, say $g^{j}\left(T, x_{T}\right)$, at maturity / exercise time $T$. Therefore, the security prices, which are expressed as present values of their dividend streams are well defined for all finite-lived securities (with or without lump-sum dividend payments). This is particularly true for bonds, options and other derivative securities.

For long-lived securities, such as the market portfolio, sufficient conditions to ensure well-defined security prices are summarized by (A0) below. 
(A0) For each long-lived security $j$, the discounted dividend streams are progressively $\mathbb{F}_{t}$-measurable and integrable, and take finite present values almost surely; i.e., at all $(t, x)$,

$$
\mathbb{E}\left[\int_{t}^{\infty} e^{-\int_{t}^{s} r\left(\tau, \widehat{x}_{\tau-}\right) d \tau} \delta^{j}\left(s, \widehat{x}_{s-}\right) d s \mid \widehat{x}_{t}=x\right]<\infty .
$$

It remains to prove the existence of equilibrium. We need to show that, for the security price processes given by equation (15), the representative agent chooses optimally to hold one unit of each of the risky assets, and to consume the aggregate dividend $c=\delta^{m} \in \mathbb{D}$. We have:

Theorem 10 Under assumptions $(A O)$ and $(U)$, the representative agent economy admits an equilibrium, in which the equilibrium security prices are governed by the present value (PV) pricing rule (15): ${ }^{9}$

$$
\begin{aligned}
S^{j}\left(t, x_{t}\right) & =\mathbb{E}_{t}\left[\int_{t}^{T} e^{-\int_{t}^{s} r\left(\tau, \widehat{x}_{\tau-}\right) d \tau} \delta^{j}\left(s, \widehat{x}_{s-}\right) d s+e^{-\int_{t}^{T} r\left(\tau, \widehat{x}_{\tau-}\right) d \tau} S^{j}\left(T, \widehat{x}_{T}\right)\right] \\
\forall T & \geq t \geq 0, j=1, \ldots, n .
\end{aligned}
$$

Proof. First, the representative agent is endowed with one share on each of the risky securities. In other words, the agent is endowed with one unit of the market portfolio $m$. Given the security price process (15), together with assumption (A0), the initial wealth $W_{0}$ for the agent is finite, and coincides with the time- 0 price $S_{0}^{m}$ of the market portfolio $m$ determined by equation (15). Therefore, for any admissible consumption program $c^{\prime} \equiv\left\{c^{\prime}\left(t, x_{t}\right)\right\}_{t>0} \in \mathcal{C}_{0}$, the corresponding initial cost for achieving $c^{\prime}$ cannot exceed $W_{0}=S_{0}^{m}$ following the budget feasibility condition. This reduces to the following inequality: ${ }^{10}$

$$
\mathbb{E}\left[\int_{0}^{\infty} e^{-\int_{0}^{s} r\left(\tau, \widehat{x}_{\tau-}\right) d \tau} c^{\prime}\left(s, \widehat{x}_{s-}\right) d s \mid \widehat{x}_{0}=x_{0}\right] \leq S_{0}^{m} .
$$

By assumption, the representative agent's utility function is well-defined, namely, the time- $t$ utility function $V_{t}(c), \forall t \geq 0$, as the unique solution to the Koopmans equation (5) is concave, and is monotonic increasing in $c$. Suppose that, to the contrary, at $c^{\prime}$ the agent achieves a higher utility than the utility at $c \equiv \delta^{m}$; i.e., $V_{0}\left(c^{\prime}\right)>V_{0}(c)$. Consider the real function

$$
G(\alpha) \equiv V_{0}\left(\alpha c^{\prime}+(1-\alpha) c\right)=V_{0}\left(c+\alpha\left(c^{\prime}-c\right)\right)
$$

\footnotetext{
${ }^{9}$ The conditional expectation in the expression below is understood to be conditional on $\widehat{x}_{t}=x_{t}$.

${ }^{10}$ Let $z^{\prime}$ and $W^{\prime}$ be respectively the portfolio holdings and wealth process for the admissible consumption plan $c^{\prime}$. Under the pseudo-state process $\widehat{x}$ defined above, together with the security price process governed by (15), we can show that $W^{\prime}$ satisfies the PDDE:
}

$$
\widehat{\mathcal{A}} W^{\prime}-r W^{\prime}+c^{\prime}=0 \text {. }
$$

Therefore, by the Feynman-Kac formula, the initial expenditure $W_{0}^{\prime}$, which is required to achieve $c^{\prime}$, is given by the left hand side of (18). 
for $\alpha \in[0,1] . G(\cdot)$ is concave and increasing in $\alpha$. This implies: $G^{\prime}(0+) \geq$ $G(1)-G(0)$. Or, equivalently,

$$
0<V_{0}\left(c^{\prime}\right)-V_{0}(c) \leq \partial V_{0}\left(c, c^{\prime}-c\right)
$$

in which $\partial V_{0}\left(c, c^{\prime}-c\right)$ denotes the time-0 utility gradient at $c$ in the direction $c^{\prime}-c$.

Furthermore, by differentiating both sides of the Koopmans equation (5), we see that the utility gradient process $\left\{\partial V_{t}\left(c, c^{\prime}-c\right)\right\}_{t \geq 0}$ at $\left(c, c^{\prime}-c\right)$ satisfies the following equation:

$$
\begin{aligned}
& \partial V_{t}\left(c, c^{\prime}-c\right) \\
= & \frac{1}{\pi_{t}} \mathbb{E}_{t}\left[\int_{t}^{T}\left\{\pi_{s_{-}}\left(c_{s}^{\prime}-c_{s}\right)+\int_{\mathbb{R}} M_{1}\left(V_{s+}, V_{s-}\right) \pi_{s+} \partial V_{s+}\left(c, c^{\prime}-c\right) v(d u)\right\} d s\right. \\
& \left.+\pi_{T} \partial V_{T}\left(c, c^{\prime}-c\right)\right]
\end{aligned}
$$

for all $T \geq t \geq 0$. Therefore, the utility gradient satisfies equation (11) with "dividend" stream $c^{\prime}-c$. Notice also that condition (A0) is satisfied, since the infinite integration takes a finite value as $T \rightarrow \infty$. By Proposition 7, the initial utility gradient is given by

$$
\begin{aligned}
& \partial V_{0}\left(c, c^{\prime}-c\right) \\
= & \mathbb{E}\left[\int_{0}^{\infty} e^{-\int_{0}^{s} r\left(\tau, \widehat{x}_{\tau-}\right) d \tau}\left(c^{\prime}\left(s, \widehat{x}_{s-}\right)-c\left(s, \widehat{x}_{s-}\right)\right) d s \mid \widehat{x}_{0}=x_{0}\right] \\
= & \mathbb{E}\left[\int_{0}^{\infty} e^{-\int_{0}^{s} r\left(\tau, \widehat{x}_{\tau-}\right) d \tau} c^{\prime}\left(s, \widehat{x}_{s-}\right) d s \mid \widehat{x}_{0}=x_{0}\right]-S_{0}^{m} \\
\leq & 0
\end{aligned}
$$

in which the right hand side is non-positive following the budget feasibility condition (18) for $c^{\prime}$ at $t=0$. This, however, constitutes a contradiction to the inequality (19).

In conclusion, the security pricing rule given by equation (15) must support the aggregate dividend as an optimal consumption plan. In particular, security prices governed by the PV pricing rule constitute the equilibrium prices for the representative agent's economy. This ends the proof.

\section{Option Pricing: A Closed-Form Formula}

This section considers a particular parametric specification of the economy studied in the previous section. A closed-form formula for the price of aggregate equity, European call options and other derivative securities written on the aggregate equity are provided. 


\subsection{Assumptions}

In addition to assumption (A0), we assume a homothetic recursive utility with an utility generator $(f, M)$ to be such that

$$
\begin{aligned}
f\left(k c, k^{\eta} v\right) & =k^{\eta} f(c, v), \forall k>0, \forall(c, v) \in \mathbb{R}_{+} \times \mathbb{R} \\
M(x, y) & =y \phi\left(\frac{x}{y}\right)+y-x, \forall(x, y) \in \mathbb{R} \times \mathbb{R}
\end{aligned}
$$

where $\phi \in C^{2}(\mathbb{R})$ is increasing, and satisfies the following conditions: $\phi(1)=$ $\phi^{\prime \prime}(1)=0$ and $\phi^{\prime}(1)=1$; and where the parameter $\eta$ is a measure of risk aversion for "small" gambles about certainty. When $\phi(z)=z-1$, the utility function reduces to the $\mathrm{KP}$ expected utility. A further specification of $f(c, v)=$ $\frac{c^{\eta}}{\eta}-\beta v$ results in expected additive power utility function.

For the information structure, we assume that there is only one state variable, which is the aggregate dividend $x_{t} \equiv \delta_{t}$. The dividend process $\delta=\left\{\delta_{t}\right\}_{t \geq 0}$ is assumed to follow a jump diffusion process

$$
\frac{d \delta_{t}}{\delta_{t-}}=b d t+a d B_{t}+\int_{0}^{\infty}(u-1) v(d t, d u)
$$

with initial condition $\delta_{0}>0$, and with constant coefficients $b$ and $a$. Here, $u$ takes positive value only and admits a natural interpretation: when a jump occurs at time $t$ with size $u$, the aggregate dividend rate jumps from $\delta_{t-}$ to $\delta_{t+}=\delta_{t-}+(u-1) \delta_{t-}=u \delta_{t-}$.

We impose the following joint restrictions on the utility generator and the state process:

(A1) Let $Q(z)=b z+\frac{z(z-1)}{2} a^{2}, z \in \mathbb{R}$. Assume that $\int_{0}^{\infty} \phi\left(u^{\eta}\right) \nu(d u)<\infty$ is integrable, and that there is a unique $k$, which has the same sign as $\eta$, solving

$$
\frac{f(1, k)}{k}+Q(\eta)+\int_{0}^{\infty} \phi\left(u^{\eta}\right) \nu(d u)=0
$$

with $f_{v}(1, k)+Q(\eta)+\int_{0}^{\infty} \phi\left(u^{\eta}\right) \nu(d u)<0$.

(A2) There exists a $x^{*}<-1$ such that, for all $x \in\left(x^{*}, 0\right]$,

$$
\int_{0}^{\infty} \phi^{\prime}\left(u^{\eta}\right) u^{\eta-x-1} \nu(d u)<\infty
$$

Condition (A1) is to ensure that the value function at the aggregate dividend as a solution to the Koopmans equation (5) is well-defined. The value function is, in fact, given by $V_{t}=k \delta_{t}^{\eta}$. Condition (A2) is a technical integrability condition to ensure the pseudo-Lévy measure given in equation (25) below to admit a finite moment up to order $-x^{*}$. Condition (A2) is satisfied, for instance, when $\phi(\cdot)$ has bounded first order derivatives, and when the original Lévy measure $\nu(\cdot)$ is with a finite moment of order $\sigma$, for all $\sigma \in\left[\eta-1, \eta-x^{*}-1\right)$. 


\subsection{Derivative Securities on Aggregate Equity}

We consider derivative securities on the aggregate equity with a lump-sum payoff $G\left(S_{T}\right)$ at maturity date $T$. For the European call option with strike price $K$, we have $g(S)=(S-K)^{+}$. The analysis below can be applied for more general situations when a derivative security has also a continuum dividend stream, and/or with maturity given by pre-specified random stopping time $T$, such as the case for the American options.

We start with the following observations, the proofs of which are omitted because of their simplicity.

1. The time- $t$ utility at the aggregate consumption $\delta$ is $V_{t}(\delta)=k \delta_{t}^{\eta}$, which solves the Koopmans equation (5). Here, $k$ is the unique solution to equation (23).

2. The equilibrium pseudo state price $\pi$ is given by

$$
\begin{gathered}
\pi_{t}=f_{c}(1, k) e^{\rho t} \delta_{t}^{\eta-1} \\
\text { with } \rho=f_{v}(1, k)+\int_{0}^{\infty}\left[1+\phi\left(u^{\eta}\right)-u^{\eta} \phi^{\prime}\left(u^{\eta}\right)\right] \nu(d u) .
\end{gathered}
$$

3. The equilibrium interest rate is constant:

$$
r=-\left\{f_{v}(1, k)+Q(\eta-1)+\int_{0}^{\infty}\left[\phi\left(u^{\eta}\right)+\left(u^{\eta-1}-u^{\eta}\right) \phi^{\prime}\left(u^{\eta}\right)\right] \nu(d u)\right\} .
$$

4. The pseudo-state process $\widehat{x}_{t}=\widehat{\delta}_{t}$ follows the motion

$$
\frac{d \widehat{\delta}_{t}}{\widehat{\delta}_{t-}}=\left(b+(\eta-1) a^{2}\right) d t+a d \widehat{B}_{t}+\int_{0}^{\infty}(u-1) \widehat{v}(d t, d u)
$$

with $\widehat{\delta}_{0}=\delta_{0}$. The corresponding Lévy measure for the pseudo Poisson random measure $\widehat{v}(\cdot, \cdot)$ is given by

$$
\widehat{\nu}(d u)=\phi^{\prime}\left(u^{\eta}\right) u^{\eta-1} \nu(d u) .
$$

5. In equilibrium, the aggregate equity is with a constant dividend-price ratio $\kappa=\frac{f_{c}(1, k)}{\eta k}>0$ so that $S_{t}=\kappa^{-1} \delta_{t}$. Consequentially, equity price process $S$ follows the same motion as the dividend rates process $\delta$ (except the initial condition).

6. The time- $t$ price $S_{t}^{T, g}$ for the derivative security $\left(T, g\left(S_{T}\right)\right)$ is given by

$$
S^{T, g}\left(t, S_{t}\right)=e^{-r(T-t)} \mathbb{E}\left[g\left(\widehat{S}_{T}\right) \mid \widehat{S}_{t}=S_{t}\right] .
$$

Denote by $\mathcal{K}$ the complex plane. For each $s \in K$, we write $s=\operatorname{Re}(s)+$ $i \operatorname{Im}(s)$ with real component $\operatorname{Re}(s)$, complex component $\operatorname{Im}(s)$, along with the imaginary number $i$ satisfying $i^{2}=-1$. 
For the prescribed pseudo-state process, we consider a complex function $\Theta(\cdot): K \rightarrow K$ defined by setting ${ }^{11}$

$$
\begin{aligned}
\ln \Theta(s) & =-\left[b+(\eta-1.5) a^{2}\right] s+.5 a^{2} s^{2}+\int_{0}^{\infty}\left(u^{-s}-1\right) \widehat{\nu}(d u) \\
& =(\kappa-r) s+\frac{a^{2} s(s+1)}{2}+\int_{0}^{\infty}\left[u^{-s}+(u-1) s-1\right] \widehat{\nu}(d u)(27)
\end{aligned}
$$

As illustrated in Appendix B, $\Theta(\cdot)$ is the characteristic function for the instantaneous pseudo growth rate $\frac{1}{T}\left(\ln \widehat{S}_{T}-\ln S_{0}\right)$. We may thus refer it to be the pseudo-m.g.f. for the aggregate equity. Under assumption (A2), the pseudom.g.f. $\Theta(s)$ is well defined on $\operatorname{Re}(s) \in\left(x^{*}, 0\right)$.

For any arbitrary complex function, $F(s)$, its Laplace inverse transform $L^{-1}\{F(s)\}(x)$ is a linear operator that is defined by setting

$$
\mathcal{L}^{-1}\{F(s)\}(x)=\frac{1}{2 \pi i} \int_{\sigma-i \infty}^{\sigma+i \infty} e^{s x} F(s) d s
$$

whenever the improper integral on right hand side is well-defined. To compute the Laplace inverse transform, one may first compute the integral $\int_{\sigma-z i}^{\sigma+z i} e^{s x} F(s) d s$ along the vertical line $\operatorname{Re}(s)=\sigma$ with $\operatorname{Im}(s) \in[-z, z]$, then take the limit $\lim _{z \rightarrow+\infty} \int_{\sigma-z i}^{\sigma+z i} e^{s x} F(s) d s$ if it exists.

We have:

Theorem 11 Under conditions (A0), (A1) and (A2), the time-t equilibrium price for a derivative security with lump sum payment $g\left(S_{T}\right)$ at maturity date $T$ is given by

$$
S^{T, g}\left(t, S_{t}\right)=e^{-r(T-t)} \int_{\mathbb{R}} \mathcal{L}^{-1}\left\{\Theta^{T-t}(s)\right\}\left(y-\ln S_{t}\right) g\left(e^{y}\right) d y
$$

provided that the right hand side takes a finite value.

Proof. See Appendix B.

Corollary 12 Under the above listed assumptions, given equity price $S$ at time $t$, the time-t equilibrium price $C_{t}(T, S, K)$ of a European call option on the aggregate equity with exercise price $K$ and maturity date $T$ is given by

$$
C_{t}(T, S, K)=K e^{-r(T-t)} \mathcal{L}^{-1}\left\{\Phi_{T-t}(s)\right\}\left(\ln \frac{K}{S}\right)
$$

where $\Phi_{T-t}(s)=\frac{\Theta^{T-t}(s)}{s(s+1)}, \operatorname{Re}(s) \in\left(x^{*},-1\right)$.

\footnotetext{
${ }^{11}$ It is easy to verify that, the difference between the equilibrium interest rates and the dividend-price ratio admits the following expression:

$$
r-\kappa=b+(\eta-1) a^{2}+\int_{\mathbb{R}}(u-1) \widehat{\nu}(d u)
$$
}




\section{Proof. See Appendix B.}

Remark 13 Function $\Phi_{T-t}(s)=\frac{\Theta^{T-t}(s)}{s(s+1)}$ is referred to as "transmitting function", which admits an one-to-one correspondence with the pricing kernel implied by the no-arbitrage theorem. In Appendix B, it is shown that $\mathcal{L}^{-1}\left\{\Phi_{T-t}\right\}(\cdot)$ is well-defined for all $t \in[0, T]$. Moreover, it takes non-negative finite values, and is infinitely continuously differentiable. For the case when $\widehat{\nu}(\cdot)$ has finite second moment, one may set $\sigma=-2$ to compute the Laplace inverse transformation to the transmitting function $\Phi_{T-t}(s)$ to obtain the option price.

Remark 14 The prices for European put options can be deduced from the "putcall-futures parity". We have:

$$
P_{t}(T, S, K)=C_{t}(T, S, K)+e^{-r(T-t)}\left(K-F_{t, T}\right)
$$

with futures price $F_{t, T}$ to be given by $e^{(r-\kappa)(T-t)} S_{t}$.

Remark 15 The effect of preference specifications on the prices of derivative securities is mainly reflected through the pseudo-m.g.f. $\Theta$ as well as the transmitting function $\Phi$, taking as given the risk free interest rate $r$. For example, Ma (1992, 2005) proved that the underlying pseudo-m.g.f. corresponding to a non-expected utility function cannot be recovered by any expected utility function specification except when the uncertainty is either driven by a pure Brownian motion (Black-Scholes 1973), or purely driven by a Poisson process with a fixed jump size (Cox and Ross 1976). Therefore, we conclude that these two classes of utility functions are observational distinguishable since they generate different pricing rules for option prices. ${ }^{12}$

\subsection{Option Pricing with Pure Brownian Information}

Here, we want to derive the Black-Scholes option pricing formula as a special case of the expression (30) by specifying $\nu(\cdot) \equiv 0$, which corresponds to the pure Brownian information as assumed in the original work of Black and Scholes (1973).

Without loss of generality, we consider the $t=0$ price for the option. We have $\Theta(s)=e^{-(r-\kappa) s+.5 a^{2} s(s+1)}$. We have

$$
\begin{aligned}
\mathcal{L}^{-1}\left\{\Phi_{T}(s)\right\}(z) & =\mathcal{L}^{-1}\left\{\Theta^{T}(s)\left(\frac{1}{s}-\frac{1}{s+1}\right)\right\}(z) \\
& =\int_{z}^{\infty} \mathcal{L}^{-1}\left\{\Theta^{T}(s)\right\}(x)\left(e^{x-z}-1\right) d x
\end{aligned}
$$

\footnotetext{
${ }^{12}$ Notice that the aggregate equity price $S$ follows the same stochastic process as the aggregate dividend rate $\delta$, except that the initial condition for $S$ is given by $S_{0}=\kappa \delta_{0}$. The utility specification affects the aggregate equity only through the constant equity-dividend ratio $\kappa$. Therefore, one cannot distinguish between recursive utility and expected discounted utility merely by observing the prices of the aggregate equity. The same observation holds true for bond prices since the interest rate in this economy is constant over time.
} 
following the convolution and lag principles for the bilateral Laplace inverse transformation and the fact that, when $\operatorname{Re}(s)<0$, it holds true that

$$
\mathcal{L}^{-1}\left\{\frac{1}{s}\right\}(x)=\left\{\begin{array}{l}
0, \text { if } x>0 \\
-1, \text { otherwise }
\end{array}\right.
$$

Notice also that $\Theta^{T}(s)$ is the moment generating function of a normal distribution function with mean and variance respectively given by $\left(r-\kappa-.5 a^{2}\right) T$ and $a^{2} T$. We have,

$$
\mathcal{L}^{-1}\left\{\Theta^{T}(s)\right\}(x)=\frac{1}{\sqrt{2 \pi T} a} e^{-.5\left(\frac{x-\left(r-\kappa-.5 a^{2}\right) T}{a \sqrt{T}}\right)^{2}} .
$$

Substitute (32) into (31), and compute the integral, we have

$$
\mathcal{L}^{-1}\left\{\Phi_{T}(s)\right\}(z)=e^{(r-\kappa) T} N\left(d_{1}[z]\right) e^{-z}-N\left(d_{2}[z]\right)
$$

where $d_{1}[z]=\frac{\left(r-\kappa+.5 a^{2}\right) T-z}{a \sqrt{T}}, d_{2}[z]=\frac{\left(r-\kappa-.5 a^{2}\right) T-z}{a \sqrt{T}}$ and $N(\cdot)$ is the standard normal distribution function.

Substitute the above expression (33) into our option pricing formula (30) and set $z=\ln \frac{K}{S}$, we obtain the famous Black-Scholes option pricing formula with constant dividend-equity ratio $\kappa$ :

$$
C_{0}(T, S, K)=e^{-\kappa T} N\left(d_{1}[z]\right) S-K e^{-r T} N\left(d_{2}[z]\right)
$$

\subsection{Naik-Lee's Option Pricing Formula Revisited}

Let $\lambda_{0}=\nu\left(\mathbb{R}_{+}\right)$and $\widehat{\lambda}_{0}=\widehat{\nu}\left(\mathbb{R}_{+}\right)$be positive, which are interpreted as "jump intensities" associated with the underlying Lévy measures respectively. They are so named because $\lambda_{0}$ and $\widehat{\lambda}_{0}$ measure respectively the total intensities of jumps of all possible sizes.

Let $\Pi(\cdot)=\nu(\cdot) / \lambda_{0}$ and $\widehat{\Pi}(\cdot)=\widehat{\nu}(\cdot) / \widehat{\lambda}_{0}$ which admit natural interpretation as the jump size distributions for the corresponding Lévy processes. We write

$$
\Phi_{T}(s)=e^{-\widehat{\lambda}_{0} T} \Phi_{0, T}(s) e^{\widehat{\lambda}_{0} T \int_{0}^{\infty} u^{-s} \widehat{\Pi}(d u)}
$$

with

$$
\Phi_{0, T}(s)=\frac{e^{-\left[b+(\eta-1.5) a^{2}\right] T s+.5 T a^{2} s^{2}}}{s(s+1)} .
$$

corresponding to the transmitting function in absence of jump risk. Applying Taylor's expansion, we have

$$
\begin{aligned}
& e^{\widehat{\lambda}_{0} T \int_{0}^{\infty} u^{-s} \widehat{\Pi}(d u)} \\
= & \sum_{n=0}^{\infty} \frac{\left(\widehat{\lambda}_{0} T\right)^{n}}{n !}\left[\int_{0}^{\infty} u^{-s} \widehat{\Pi}(d u)\right]^{n} \\
= & \sum_{n=0}^{\infty} \frac{\left(\widehat{\lambda}_{0} T\right)^{n}}{n !} \int_{0}^{\infty} \cdots \int_{0}^{\infty} \prod_{i=0}^{n-1} u_{i}^{-s} \widehat{\Pi}\left(d u_{i}\right) .
\end{aligned}
$$


Therefore,

$$
\begin{aligned}
& \mathcal{L}^{-1}\left\{\Phi_{T}(s)\right\}(z) \\
= & \sum_{n=0}^{\infty} \frac{e^{-\widehat{\lambda}_{0} T}\left(\widehat{\lambda}_{0} T\right)^{n}}{n !} \int_{0}^{\infty} \cdots \int_{0}^{\infty} \mathcal{L}^{-1}\left\{\Phi_{0, T}(s) e^{-s \sum_{i=0}^{n-1} \ln u_{i}}\right\}(z) \prod_{i=0}^{n-1} \widehat{\Pi}\left(d u_{i}\right) \\
= & \sum_{n=0}^{\infty} \frac{e^{-\widehat{\lambda}_{0} T}\left(\widehat{\lambda}_{0} T\right)^{n}}{n !} \int_{0}^{\infty} \cdots \int_{0}^{\infty} \mathcal{L}^{-1}\left\{\Phi_{0, T}(s)\right\}\left(z-\sum_{i=0}^{n-1} \ln u_{i}\right) \prod_{i=0}^{n-1} \widehat{\Pi}\left(d u_{i}\right)
\end{aligned}
$$

where the first equality follows by the linearity of the Laplace inverse transformation, and the last equality follows by the definition of the transformation.

Let $\left\{u_{i}\right\}_{i=0}^{n-1}$ be i.i.d. random variables with distribution function $\widehat{\Pi}(\cdot)$. Denote by $\widehat{\Pi}_{\ln }^{n}(\cdot)$ the corresponding probability function for $\sum_{i=0}^{n-1} \ln u_{i}$. We have,

$$
\begin{aligned}
& \mathcal{L}^{-1}\left\{\Phi_{T}(s)\right\}(z) \\
= & \sum_{n=0}^{\infty} \frac{e^{-\widehat{\lambda}_{0} T}\left(\widehat{\lambda}_{0} T\right)^{n}}{n !} \int_{-\infty}^{\infty} \mathcal{L}^{-1}\left\{\Phi_{0, T}(s)\right\}(z-y) d \widehat{\Pi}_{\ln }^{n}(y) .
\end{aligned}
$$

Now, we consider the expected utility specification with $\phi^{\prime}(\cdot) \equiv 1$, and assume that the jump size follows a log-normal distribution function. This corresponds to the case studied by Naik and Lee (1990).

Suppose that $\Pi(\cdot)$, the log-normal distribution function, has parameters summarized by $\left(\mu, \Sigma^{2}\right)$; i.e., $\ln u \sim N\left(\mu, \Sigma^{2}\right)$. The corresponding distribution function $\widehat{\Pi}(\cdot)$ is also a distribution function for a log-normal random variable. In particular, $\widehat{\Pi}(\cdot)$ has parameters respectively given by $\widehat{\mu}=\mu+(\eta-1) \Sigma^{2}$ and $\widehat{\Sigma}^{2}=\Sigma^{2}$. Moreover, $\widehat{\lambda}_{0}=\lambda_{0} e^{(\eta-1) \mu+.5(\eta-1)^{2} \Sigma^{2}}$. Hence, $\widehat{\Pi}_{\mathrm{ln}}^{n}(\cdot) \sim N\left(n \widehat{\mu}, n \Sigma^{2}\right)$. Substitute this expression for $\widehat{\Pi}_{\mathrm{ln}}^{n}(d y)$ into equation (36), and compute the integral to deduce

$$
\begin{aligned}
& \mathcal{L}^{-1}\left\{\Phi_{T}(s)\right\}(z) \\
= & \sum_{n=0}^{\infty} \frac{e^{-\widehat{\lambda}_{0} T}\left(\widehat{\lambda}_{0} T\right)^{n}}{n !}\left\{e^{\left[b+(\eta-1) a^{2}\right] T} e^{n\left[\eta \mu+\frac{(\eta \Sigma)^{2}}{2}\right]} N\left(d_{1 n}[z]\right) S\right. \\
& \left.-e^{n\left[(\eta-1) \mu+\frac{[(\eta-1) \Sigma]^{2}}{2}\right]} N\left(d_{2 n}[z]\right)\right\}
\end{aligned}
$$

where $d_{1 n}[z]=d_{2 n}[z]+\Sigma_{n}, d_{2 n}[z]=\frac{\left[b+(\eta-1.5) a^{2}\right] T+n\left[\mu+(\eta-1) \Sigma^{2}\right]-z}{\Sigma_{n}}$ and $\Sigma_{n}^{2}=$ $a^{2} T+n \Sigma^{2}$. Substituting this into the right hand side of equation (30), we obtain Naik-Lee's option pricing formula.

It reduces to Merton's (1976) formula by setting $\eta=1$. A further specification of $a^{2}=0$ and $\Sigma^{2}=0$ leads to the Cox-Ross (1976) formula. 


\subsection{Jump Risk and Moneyness Biases}

A fairly large body of empirical evidence is available which suggests that the Black-Scholes model mis-prices the underlying options. With index options in particular, as reported by Bailey and Stulz (1989), the Black-Scholes formula tends to underprice the in-the-money options and overprice the out-of-money options. One explanation toward such moneyness biases associated with the Black-Scholes option pricing formula is due to its ignorant in modelling the jump risk associated with the stock price movement. The exercise carried out below shows the (theoretical) potential usefulness of introducing the jump risk as an explanation toward the moneyness biases associated with the Black-Scholes model.

Let $C_{0}^{\mathrm{BS}}(T, S, K)$ denote the right hand side of (34), which is the time-0 option price given by the Black-Scholes formula. Here, we restrict the interest rate $r$, dividend-equity ratio $\kappa^{-1}$ and volatility $a$ to be the same as in the pure diffusion case for (30). From equation (35), we see that the time-0 price $C_{0}(T, S, K)$ for the European call option by introducing the jump risk can be expressed as

$$
\begin{aligned}
& C_{0}(T, S, K)-C_{0}^{\mathrm{BS}}(T, S, K) \\
= & \left(e^{-\widehat{\lambda}_{0} T}-1\right) C_{0}^{\mathrm{BS}}(T, S, K) \\
& +\sum_{n=1}^{\infty} \frac{e^{-\widehat{\lambda}_{0} T}\left(\widehat{\lambda}_{0} T\right)^{n}}{n !} \int \cdots \int C_{0}^{\mathrm{BS}}\left(T, S \prod_{i=1}^{n} u_{i}, K\right) \prod_{i=1}^{n} \widehat{\Pi}\left(d u_{i}\right) \\
= & \widehat{\lambda}_{0} T \int_{0}^{\infty} \Delta C_{0}^{\mathrm{BS}}(T, u \mathrm{~S}, K) \widehat{\Pi}(d u)+o\left(\lambda_{0} T\right)
\end{aligned}
$$

where

$$
\Delta C_{0}^{\mathrm{BS}}(T, u \mathrm{~S}, K)=C_{0}^{\mathrm{BS}}(T, u \mathrm{~S}, K)-C_{0}^{\mathrm{BS}}(T, S, K)
$$

is the difference between the Black-Scholes price with a modified initial stock price, given by $u \mathrm{~S}, u \in \mathbb{R}_{+}$, and the Black-Scholes price with initial stock price $S$. Therefore, the biases associated with the Black-Scholes model are of magnitude $O\left(\lambda_{0} T\right)$ when the jump intensity $\left(\lambda_{0}\right)$ and the time-to-maturity $(T)$ are small.

From equation (38), we see that the sign of the bias associated with the Black-Scholes model is determined by an "weighted average" differences between the Black-Scholes prices with the modified initial stock prices $u \mathrm{~S}, u \in \mathbb{R}_{+}$, and the Black-Scholes price with the true initial stock price $S$. Recall that, option price given by the Black-Scholes formula increases with the initial stock price; that is,

$$
\operatorname{sign}\left(\Delta C_{0}^{\mathrm{BS}}(T, u \mathrm{~S}, K)\right)= \begin{cases}+ & \text { if } u>1 \\ - & \text { if } u<1\end{cases}
$$

Therefore, the jump size distribution function $\widehat{\Pi}(\cdot)$ corresponding to the pseudo-state process is crucial to determine if the Black-Scholes model overprices or underprices the underlying options. In particular, from equation (38), 
we see that, in a bear market when the stock index is more likely to decline, ${ }^{13}$ the Black-Scholes model will tend to overprice the options; and similarly, in a bull market when stock index is more likely to expand, the Black-Scholes model will tend to underprice the option. These are particularly true for options with short time-to-maturities, and when the jump intensities are small.

Let $\xi \equiv \frac{S}{K}$ denote the price-exercise ratio for the call option. When $\xi>1$ the option is said to be in-the-money; when $\xi<1$ it is out of money, and when $\xi=1$ it is referred to as the at-the-money option. Notice that $C_{0}^{\mathrm{BS}}(T, \mathrm{~S}, K) \equiv$ $K C_{0}^{\mathrm{BS}}(T, \xi, 1)$, and that

$$
\operatorname{sign}\left(\int_{0}^{\infty} \Delta C_{0}^{\mathrm{BS}}(T, u \mathrm{~S}, K) \widehat{\Pi}(d u)\right)=\operatorname{sign}\left(\int_{0}^{\infty} \Delta C_{0}^{\mathrm{BS}}(T, u \xi, 1) \widehat{\Pi}(d u)\right) .
$$

Therefore, the sign of the bias produced by the Black-Scholes model is jointly determined by the price-exercise ratio $\xi$ and the (modified) jump size distribution function $\widehat{\Pi}(\cdot)$. In order to generate a pattern that is consistent with Bailey and Stulz (1989)'s findings, we assume that, for at-the-money options, the following equality holds: ${ }^{14}$

$$
\int_{0}^{\infty} C_{0}^{\mathrm{BS}}(T, u, 1) \widehat{\Pi}(d u)=C_{0}^{\mathrm{BS}}(T, 1,1)+o(1) .
$$

Given this, the condition under which $\int_{0}^{\infty} \Delta C_{0}^{\mathrm{BS}}(T, u \xi, 1) \widehat{\Pi}(d u)$ changes sign from negative to positive as $\xi$ increases, at a neighborhood of $\xi=1$, becomes

$$
\int_{0}^{\infty} N\left(d_{1}[-\ln u]\right) u \widehat{\Pi}(d u)>N\left(d_{1}[0]\right)
$$

noticing that $\frac{\partial C_{0}^{\mathrm{BS}}(T, x, 1)}{\partial x}=e^{-\kappa T} N\left(d_{1}[-\ln x]\right)$. Or, in words, under assumptions (39) and (40), option pricing formula (30) produces higher values for inthe-money options and lower values for out-of-money options, relative to the Black-Scholes model at a neighborhood of $\xi=1$. Of course, this holds only when the time to maturity and jump intensity are small.

Numerical experiments were carried out by Ma and Vetzal (1995) which show how option pricing formula (30) could produce predictions that are consistent with Bailey and Stulz (1989)'s findings.

\section{Concluding Remarks}

In summary, the option pricing formula resulted in this paper is attractive for both its mathematical simplicity and for its generality in comparison with those in the literature. The utility function can take a general form and so can the

\footnotetext{
${ }^{13}$ This corresponds to the situation when the distribution function $\widehat{\Pi}(\cdot)$ has a "dominating" density for downward jumps with $u \in(0,1)$ relative to the upward density.

${ }^{14}$ Under this assumption, the difference between the theoretical price with jump risk to the Black-Scholes price for at-the-money options is of order $o\left(\lambda_{0} T\right)$.
} 
distribution function for the size of the jumps. More importantly, in contrast to the option pricing formula derived with the expected utility specification, our option pricing formula with a general recursive utility function disentangles the effects on asset pricing of risk aversion, intertemporal substitution and the other aspects of attitudes toward risk and uncertainty in the representative agent's preference specification.

The derived option pricing formula is also potentially useful in addressing the empirical biases associated with the Black-Scholes and other formulae to the observed market data. Ma and Vetzal (1995), and Asea, Ma and Ncube (1997) each contain some numerical examples which demonstrate the usefulness of our model in addressing these issues.

\section{A A Generalized Feynman-Kac Formula}

Let $C^{1,2}\left([0, T] \times \mathbb{R}^{n}\right)$ be the space of continuous functions with respectively first and second order continuous derivatives in $t \in(0, T)$ and $x \in \mathbb{R}^{n}$. To prove the generalized Feynman-Kac formula, we need to introduce first the so-called strong smoothness condition:

Definition 16 A function $f \in C^{1,2}\left([0, T] \times \mathbb{R}^{n}\right)$ is said to satisfy the Strong Smoothness Condition if $f$ is uniformly bounded with uniform bounded first order and second order (partial) derivatives with respect to $x$.

For the given jump-diffussion state process $\left\{x_{t}\right\}$ with coefficients $\{b, a, l\}$, we may define infinitesimal generator $\mathcal{A}$ on $h \in C^{1,2}\left(\mathbb{R}_{+} \times \mathbb{R}^{m}\right)$ for the state process by setting

$$
\mathcal{A} h(t, x) \equiv \mathcal{D} h(t, x)+\int_{\mathbb{R}}[h(t, x+l(t, x, u))-h(t, x)] \nu(d u)
$$

in which $\mathcal{D}$ is the diffusion operator induced by the diffusion components $\{b, a\}$ of the state process $x$.

Theorem 17 Let $u, \rho \in C^{1,2}\left([0, T] \times \mathbb{R}^{m}\right)$, and $g_{T} \in C^{2}\left(\mathbb{R}^{m}\right)$ to satisfy the strong smoothness condition. Let $x_{t}$ be the state variable that follows the joint Poisson and Brownian motion described in Section 2. For all $(t, x) \in[0, T] \times \mathbb{R}^{m}$,

let

$$
C(t, x)=\mathbb{E}\left[\int_{t}^{T} e^{\int_{t}^{s} \rho\left(\tau, x_{\tau-}\right) d \tau} u\left(s, x_{s-}\right) d s+e^{\int_{t}^{T} \rho\left(\tau, x_{\tau-}\right) d \tau} g_{T}\left(x_{T}\right) \mid x_{t}=x\right] .
$$

Then, $C$ is in $C^{1,2}\left((0, T) \times \mathbb{R}^{m}\right)$ and solves uniquely the following partial differential difference equation:

$$
\mathcal{A} C(t, x)+\rho(t, x) C(t, x)+u(t, x)=0
$$

with boundary condition $C(T, x)=g_{T}(x)$. 
Remark 18 As a special case, when $x_{t}$ follows a diffusion process, the smoothness condition can be weakened by instead requiring the corresponding first and second order derivatives to satisfy a polynomial growth condition in $x$. See Duffie (1988, pp.226), Karatzas and Shreve (1988, Theorem 7.6), and Krylov (1980, Theorem 2.9.10).

Proof of Theorem 17. As a special case, when $\rho=u \equiv 0$, a proof to the first part of the theorem is documented in Gihman and Skorohod (1972, Chapter 2, Section 9, Theorem 4). Here, we want to show that the general case with $\rho, u \neq 0$ can be actually transformed into the case studied by Gihman and Skorohod (1972), and by making use of the findings reported there.

First, we can show that $C \in C^{1,2}\left((0, T) \times \mathbb{R}^{m}\right)$, and that it satisfies a polynomial growth condition. These actually follow the same proofs as in Gihman and Skorohod (1972, Part II, Chapter 2, Section 9, Lemmas 2 and 3, and Remark 4). The details are thus omitted.

Consider the following extended state vector $x^{\prime} \equiv\left[x^{0}, x^{1}, x\right]$ with $x_{t}^{0} \equiv$ $\int_{0}^{t} \rho\left(s, x_{s-}\right) d s$ and $x_{t}^{1} \equiv \int_{0}^{t} e^{x_{s-}^{0}} u\left(s, x_{s-}\right) d s$ for all $t \in[0, T]$. Let $g_{T}^{0}\left(x^{\prime}\right) \equiv$ $x^{1}+e^{x^{0}} g_{T}(x)$ and $C^{0}\left(t, x^{\prime}\right) \equiv \mathbb{E}\left[g_{T}^{0}\left(x_{T}^{\prime}\right) \mid x_{t}^{\prime}=x^{\prime}\right]$ for all $t \in[0, T], x^{\prime} \in \mathbb{R}^{m+2}$. We have $C^{0}\left(t, x_{t}^{\prime}\right) \equiv x_{t}^{1}+e^{x_{t}^{0}} C\left(t, x_{t}\right)$ for all $t \in[0, T]$. The function $g_{T}^{0} \in$ $C^{2}\left(\mathbb{R}^{m+2}\right)$ satisfies obviously the strong smoothness condition. Therefore, by applying Gihman and Skorohod (1972, Chapter 2, Section 9, Theorem 4), we have: $\mathcal{A}^{\prime} C^{0}\left(t, x^{\prime}\right)=0$ for all $\left(t, x^{\prime}\right)$ with boundary condition $C^{0}\left(T, x^{\prime}\right)=g_{T}^{0}\left(x^{\prime}\right)$. Here, $\mathcal{A}^{\prime}$ is the infinitesimal generator for the extended state process $\left\{x_{t}^{\prime}\right\}$. This reduces to the partial differential and difference equation (43) by noting that, for all $x^{\prime} \equiv\left[x^{0}, x^{1}, x\right]$,

$$
\mathcal{A}^{\prime} C^{0}\left(t, x^{\prime}\right)=\{\mathcal{A} C(t, x)+\rho(t, x) C(t, x)+u(t, x)\} e^{x_{0}} .
$$

It remains to show that $C$ given by (42) is the unique solution to equation (43) with boundary condition $C(T, x)=g_{T}(x)$. Suppose, to the contrary, that the equation (43) has another classical solution $C^{\prime} \in C^{1,2}\left((0, T) \times \mathbb{R}^{m}\right)$ that satisfies a polynomial growth condition, and the boundary condition. Let $\epsilon(t, x)=C(t, x)-C^{\prime}(t, x),(t, x) \in[0, T] \times \mathbb{R}^{m}$. We have,

$$
\mathcal{A} \epsilon(t, x)+\rho(t, x) \epsilon(t, x)=0, \forall(t, x) \in(0, T) \times \mathbb{R}^{m}
$$

with $\epsilon(T, x) \equiv 0$. Consider the process $\left\{y_{t} \equiv e^{\int_{0}^{t} \rho\left(s, x_{s-}\right) d s} \epsilon\left(t, x_{t}\right)\right\}_{t \in[0, T]}$. Applying Itô's Lemma to $\left\{y_{t}\right\}_{t \in[0, T]}$, we have

$$
y_{T}-y_{t}=\int_{t}^{T} \mathcal{D} y_{s-} d s+\int_{t}^{T} \sigma_{y s-} d B_{s}+\int_{t}^{T} \int_{\mathbb{R}}\left(y_{s+}-y_{s-}\right) v(d t, d u)
$$

for all $t \in[0, T]$. Note that $y_{T}=0$, and also that $\left\{y_{t}\right\}_{t \in[0, T]}$ is measurable and square integrable following from the polynomial growth conditions on $C, C^{\prime}$ and the strong smoothness condition on $\rho$. Taking the expectation on both sides of 
the above equality, we have

$$
\begin{aligned}
y_{t} & =-\mathbb{E}_{t}\left[\int_{t}^{T} \mathcal{A} y_{s-} d s\right] \\
& =-\mathbb{E}_{t}\left[\int_{t}^{T} e^{\int_{0}^{s} \rho\left(s, x_{s-}\right) d s}\left\{\mathcal{A} \epsilon_{s-}+\rho\left(s, x_{s-}\right) \epsilon\left(s, x_{s-}\right)\right\} d s\right] \\
& =0
\end{aligned}
$$

with $x_{t}=x$. This implies $\epsilon(t, x)=0$ for all $(t, x) \in[0, T] \times \mathbb{R}^{m}$. Therefore, we conclude $C=C^{\prime}$ as desired.

Remark 19 The generalized Feynman-Kac formula applies also to the case when the Lévy measure $\nu(\cdot)$ is time-varying and state-dependent. The supporting arguments for this can be found in Gihman and Skorohod (1972, pp. 245-247).

Remark 20 The strong smoothness condition can be relaxed. For example, if a sequence $\left(\rho_{n}, u_{n}, g_{n}\right) \rightarrow\left(\rho, u, g_{T}\right)$ converges uniformly as $n \rightarrow \infty$ (of course, the convergence is in the sup-norm), and each $\left(\rho_{n}, u_{n}, g_{n}\right)$ satisfies the strong smoothness condition, with solution $C_{n}$ given by the Feynman-Kac formula, then we expect that the solution $C$ for $\left(\rho, u, g_{T}\right)$ exists, and satisfies also the Feynman-Kac formula following the uniform convergence theorem for integration. For many applications, we may find the smoothness condition violated. For example, the maturity payoff function $g_{T}$ specified for a European call option obviously violates the strong smoothness condition. Nevertheless, the FeynmanKac formula still applies to this situation, following the uniform convergence argument.

Proof of Proposition 7. For any arbitrary security $j$, consider the normalized equilibrium price $Z^{j}(t, x)=\pi(t, x) S^{j}(t, x)$. Applying the generalized Feynman-Kac formula to equation (11), we obtain

$$
\mathcal{A} Z^{j}(t, x)+\int_{\mathbb{R}} M_{1+}(t, x, u) Z^{j}(t, x+l(t, x, u)) \nu(d u)+\pi(t, x) \delta^{j}(t, x)=0
$$

with $Z^{j}(T, x)=\pi(T, x) S^{j}(T, x)$, where $\mathcal{A}$ is the "infinitesimal generator" induced by jump-diffussion state process $x$. This partial differential difference equation for $Z^{j}(t, x)$ can be alternatively expressed in terms of $S^{j}(t, x)$. That is,

$$
\widehat{\mathcal{A}} S^{j}(t, x)-r(t, x) S^{j}(t, x)+\delta^{j}(t, x)=0
$$

in which $\widehat{\mathcal{A}}$ corresponds to the infinitesimal generator induced by the pseudostate process $\widehat{x}$ characterized by (14). Now, applying the generalized FeynmanKac formula to the above equation, we obtain the desired expression (15) for the price of security $j$. This ends the proof. 


\section{B Proof of Theorem 11 and Corollary 12}

The Laplace transformation is defined as the bilateral Laplace transformation, or the two sided Laplace transformation which is defined on the entire real line.

Let $\mathcal{K}$ be the complex plane. For any real function $f: \mathbb{R} \rightarrow \mathbb{R}$, the Laplace transform of $f$, denoted by $\mathcal{L}\{f(\cdot)\}(s), s \in \mathcal{K}$, is a complex-valued, function defined by

$$
\mathcal{L}\{f(\cdot)\}(s)=\int_{-\infty}^{\infty} e^{-s x} f(x) d x .
$$

The integral on the right hand side of (45) may not be well defined for some $f$ (e.g., $f(x) \equiv 1$ ). In general, this integral converges only for $s$ in a subset of $\mathcal{K}$. Actually, it can be shown that the convergence region, if not empty, must be a strip of the form $\{s \in \mathcal{K}: \operatorname{Re}(s) \in(a, b)\}$ for some real numbers $a, b \in \mathbb{R}$ (see Doetsch 1970, pp. 155).

Proof of Theorem 11: We start by computing the moment generating function for $y_{T}=\ln \widehat{S}_{T}, T>0$. The moment generating function of a random variable is defined as the Laplace transform of the underlying probability density function. We have,

Lemma 21 Under assumptions (A1) and (A2), the moment generating function for $y_{T}=\ln \widehat{S}_{T}$ exists, and is given by

$$
\mathbb{E}\left[\widehat{S}_{T}^{-s} \mid \widehat{S}_{0}=S\right]=S^{-s} \Theta^{T}(s), \operatorname{Re}(s) \in\left(x^{*},-1\right) .
$$

Proof. For any arbitrary given $s \in \mathcal{K}$ with $\operatorname{Re}(s) \in\left(x^{*},-1\right)$. Consider the complex-valued stochastic process $\left\{z_{t} \equiv \widehat{S}_{t}^{-s}\right\}_{t \geq 0}$. Applying Itô's Lemma to $z_{t}$, we have

$$
\frac{d z_{t}}{z_{t-}}=\left[-\left(b+(\eta-1.5) a^{2}\right) s+.5 a^{2} s^{2}\right] d t-a s d B_{t}+\int_{0}^{\infty}\left(u^{-s}-1\right) \widehat{v}(d t, d u)
$$

with $z_{0}=S^{-s}$. For any $t \geq 0$, let $m(t)=\mathbb{E}\left[z_{t} \mid z_{0}=S^{-s}\right]$. Multiply both sides of $(46)$ by $z_{t}$, integrate both sides from 0 to $t$, and take the expectation, to deduce

$$
\begin{aligned}
& m(t)-S^{-s} \\
= & \int_{0}^{t} m(\tau)\left\{-\left(b+(\eta-1.5) a^{2}\right) s+.5 a^{2} s^{2}+\int_{0}^{\infty}\left(u^{-s}-1\right) \widehat{\nu}(d u)\right\} d \tau \\
= & \ln \Theta(s) \int_{0}^{t} m(\tau) d \tau,
\end{aligned}
$$

where $\Theta(\cdot)$ is defined by (26), and is well-defined under assumptions (A1) and (A2). The solution to this integral equation is given by $m(t)=S^{-s} \Theta^{t}(s), \forall t \geq$ 0 . This ends the proof. 
Given the moment generating function for $y_{T}$, the underlying probability density function is uniquely determined by its inverse Laplace transform. Therefore, the time- $t$ equilibrium price $S^{T, g}(t, S)$ for the derivative security with maturity payment $G(\cdot)$ is such that

$$
\begin{aligned}
S^{T, g}(t, S) & =\mathbb{E}\left[e^{-r(T-t)} g\left(\widehat{S}_{T}\right) \mid \widehat{S}_{t}=S\right] \\
& =e^{-r(T-t)} \int_{-\infty}^{\infty} \mathcal{L}^{-1}\left\{e^{-s \ln S} \Theta^{T-t}(s)\right\}(y) g\left(e^{y}\right) d y \\
& =e^{-r(T-t)} \int_{-\infty}^{\infty} \mathcal{L}^{-1}\left\{\Theta^{T-t}(s)\right\}(y-\ln S) g\left(e^{y}\right) d y .
\end{aligned}
$$

The last equality follows from the fact that $\mathcal{L}^{-1}\left\{e^{-s x} f(s)\right\}(y)=\mathcal{L}^{-1}\{f(s)\}(y-x)$ for all $x, y$. This concludes the proof of Theorem 10 .

Proof of Corollary 12: Set $g(S)=\max \{S-X, 0\}$. Let us operate in a purely formal way, disregarding, for the moment, questions of convergence, and write

$$
\begin{aligned}
& e^{r(T-t)} C_{t}(T, S, X) \\
= & \int_{-\infty}^{\infty} \mathcal{L}^{-1}\left\{\Theta^{T-t}(s)\right\}(y-\ln S) \max \left\{e^{y}-X, 0\right\} d y \\
= & \int_{\ln X}^{\infty}\left(e^{y}-X\right)\left(\frac{1}{2 \pi i} \int_{\sigma-i \infty}^{\sigma+i \infty} \Theta^{T-t}(s) e^{(y-\ln S) s} d s\right) d y \\
= & \frac{1}{2 \pi i} \int_{\sigma-i \infty}^{\sigma+i \infty} S^{-s} \Theta^{T-t}(s)\left(\int_{\ln X}^{\infty} e^{s y}\left(e^{y}-X\right) d y\right) d s \\
= & \frac{X}{2 \pi i} \int_{\sigma-i \infty}^{\sigma+i \infty} \Phi_{T-t}(s)\left(\frac{X}{S}\right)^{s} d s \\
= & X \mathcal{L}^{-1}\left\{\Phi_{T-t}(s)\right\}\left(\ln \frac{X}{S}\right)
\end{aligned}
$$

in which $\sigma \in\left(x^{*},-1\right)$ is arbitrary. The third inequality in the above derivation involves a change of order of integrations, which needs to be justified, while all other equalities, which follow either from the definition or by simple computation, are obvious. To justify the change of order of integrations, we introduce the following useful lemmas, the proofs of which can be found in standard mathematical/real analysis textbooks, such as Apostol (1964).

Lemma 22 (Weierstrass's M-test) Let $S \subset \mathbb{R}$, and let $f(x, y)$ be defined on $[a, \infty) \times S$. Suppose the integral $\int_{a}^{b} f(x, y) d x$ exists for every $b \geq a$, and $y \in S$. If there exists a non-negative function $M(x)$ defined on $[a, \infty)$ such that the integral $\int_{a}^{\infty} M(x) d x$ converges and such that $|f(x, y)| \leq M(x), \forall x \geq a, \forall y \in S$, then the integral $\int_{a}^{\infty} f(x, y) d x$ converges uniformly on $S$.

Lemma 23 Let $f(x, y)$ be continuous on $[a, \infty) \times[c, \infty)$, and define $g(x, z) \equiv$ $\int_{c}^{z} f(x, y) d y, \forall x \geq a, z \geq c$. Suppose that 
(a) $\int_{a}^{\infty} f(x, y) d x$ converges uniformly on $y \in[c, \infty)$

(b) $\int_{a}^{\infty} g(x, z) d x$ converges uniformly on $z \in[c, \infty)$

(c) $\int_{c}^{\infty} f(x, y) d y$ converges uniformly on $x \in[a, \infty)$

(d) $\int_{a}^{\infty} \int_{c}^{\infty} f(x, y) d y d x$ converges

Then we have

$$
\int_{a}^{\infty} \int_{c}^{\infty} f(x, y) d y d x=\int_{c}^{\infty} \int_{a}^{\infty} f(x, y) d x d y
$$

Both Lemmas hold for real as well as complex functions. In particular, the lower boundaries $a$ and $c$ may take $-\infty$ since one can write $\int_{-\infty}^{\infty}=\int_{-\infty}^{0}+\int_{0}^{\infty}$ and then apply respectively the Lemma to both integrals.

Now we turn to prove the change of order of integrations to be valid. For fixed, $\sigma \in\left(x^{*},-1\right)$, let $s=\sigma+i x, x \in(-\infty, \infty)$, and let $f(x, y)=\left(e^{y}-\right.$ $X) \Theta^{T-t}(s) e^{(y-\ln S) s}, y \in[c, \infty)$ with $c=\ln X$. Similarly, define $g(x, z) \equiv$ $S^{-s} \Theta^{T-t}(s) \int_{c}^{z}\left(e^{y}-X\right) e^{y s} d y$, for all $z \geq c, x \in(-\infty, \infty)$.

The Continuity of $f$ : From the definition of $\Theta$-function, to show that $f(x, y)$ is continuous in $(x, y)$, we only need to show that $\int_{0}^{\infty} u^{-s} \phi^{\prime}\left(u^{\eta}\right) u^{\eta-1} v(d u)$ is continuous in $x$. By assumption (A2), the integral converges uniformly in $x$ since $\left|u^{-s}\right|=u^{-\sigma}$. Therefore, the integral as a function of $x$, by Apostol (1964) Theorem 14.21, is continuous since the integrand is continuous.

Condition (d): To show that the integral on the right hand side of the third equality (47) converges, first, by Lemma 22 , the integral $\int_{c}^{\infty}\left(e^{y}-X\right) e^{y s} d y$ converges uniformly to $\frac{X^{s+1}}{s(s+1)}$ when $\sigma<-1$, since $\left|\left(e^{y}-X\right) e^{y s}\right|=\left(e^{y}-X\right) e^{y \sigma}$ is positive integrable on $[c, \infty)$. Therefore, the right hand side of the third equality reduces to that of the fourth (and fifth) equality, if the latter converges. To see this, note that

$$
\begin{aligned}
& \left|\Phi_{T-t}(s)\left(\frac{X}{S}\right)^{s}\right| \\
= & \frac{\left|\Theta^{T-t}(s)\right|}{\sqrt{\left(\sigma^{2}+\sigma-x^{2}\right)^{2}+(1+2 \sigma)^{2} x^{2}}}\left(\frac{X}{S}\right)^{\sigma} \\
\leq & \frac{1}{\sigma(1+\sigma)}\left|\Theta^{T-t}(s)\right|\left(\frac{X}{S}\right)^{\sigma} \\
\leq & \frac{1}{\sigma(1+\sigma)}\left(\frac{X}{S}\right)^{\sigma} C_{0}^{T-t} e^{-\frac{(T-t) a^{2} x^{2}}{2}}
\end{aligned}
$$

since $|\Theta(s)| \leq C_{0} e^{-\frac{a^{2} x^{2}}{2}}$ for some constant $C_{0}>0$ follows from the definition of the $\Theta$-function. Setting $M(x) \equiv \frac{1}{\sigma(1+\sigma)}\left(\frac{X}{S}\right)^{\sigma} C_{0}^{(T-t)} e^{-(T-t) \frac{a^{2} x^{2}}{2}}$ for the Weierstrass's M-test, we see that the integration on the right hand side of the third 
equality converges to the right hand side of the fifth equality, which takes a finite value since $\int_{-\infty}^{\infty} M(x) d x<\infty$ for $a^{2}>0, T>t$.

Condition (a): To show $\int_{-\infty}^{\infty} f(x, y) d x$ converges uniformly on $y \in[c, \infty)$, note that

$$
\begin{array}{ll} 
& |\quad f(x, y)|=\left(e^{y}-X\right) S^{-\sigma}\left|\Theta^{T-t}(s)\right| e^{\sigma y} \\
\leq & S^{-\sigma} C_{0}^{T-t} e^{-\frac{(T-t) a^{2} x^{2}}{2}} \equiv M_{1}(x) .
\end{array}
$$

Therefore, condition (a) follows again by the Weierstrass's M-test, with test function $M_{1}(x)$.

Condition (b): To show that $\int_{-\infty}^{\infty} g(x, z) d x$ converges uniformly in $z \in$ $[c, \infty)$, we have

$$
\begin{aligned}
& |\quad g(x, z)|=S^{-\sigma}\left|\Theta^{T-t}(s)\right| \int_{c}^{z}\left(e^{y}-X\right) e^{\sigma y} d y \\
& \leq \quad C_{1} e^{-\frac{(T-t) a^{2} x^{2}}{2}} \equiv M_{2}(x)
\end{aligned}
$$

where $C_{1} \equiv \frac{X}{\sigma(1+\sigma)}\left(\frac{X}{S}\right)^{\sigma} C_{0}^{T-t}$ is a positive constant. Therefore, condition (a) follows again by the Weierstrass's M-test, with test function $M_{2}(x)$.

Condition (c): We apply the argument used to prove (a) to show that the integral $\int_{c}^{\infty} f(x, y) d y$ converges uniformly in $x \in(-\infty, \infty)$. We have

$$
\begin{aligned}
& f(x, y)\left|\leq\left(e^{y}-X\right) e^{\sigma y} S^{-\sigma}\right| \Theta^{T-t}(s) \mid \\
\leq & \left(e^{y}-X\right) e^{\sigma y} S^{-\sigma} C_{0}^{T-t} \equiv M_{3}(y) .
\end{aligned}
$$

Weierstrass's M-test applies with test function $M_{3}(y)$.

By Lemma 23, we conclude that all equalities for computing the price of the European call option are valid. In particular, the right hand side of the option pricing formula (30) takes a finite positive value. The above proof is based on the assumption of $a^{2}>0$. When $a^{2}=0$ which corresponds to the pure jumps case, the theorem still holds. The proofs are similar to those above except that we must apply Dirichlet's test for uniform convergence to verify conditions (a) and (b). The details are thus omitted.

Regarding the differentiability of the option pricing formula for $t<T$, we need to show that, each of the integrals associated with the derivatives of the integrand function with respect to $z=\ln \frac{X}{S}$ converges uniformly. This can be verified by following the same procedure as above.

\section{References}

[1] Apostol T. (1964), Mathematical Analysis. Addison-Wesley Publishing Company, Inc.

[2] Asea P., C. Ma and M. Ncube (1997), "Pricing Risk when Risk Aversion Matters", UCLA, mimeo. 
[3] Bailey W. and R.M. Stulz (1989), "The Pricing of Stock Index Options in a General Equilibrium Model", Journal of Financial and Quantitative Analysis 24, 1-12.

[4] Black, F. and M. Scholes (1973), "The Pricing of Options and Corporate Liabilities", Journal of Political Economy 81, 637-654.

[5] Brown S. and P. Dybvig (1986), "The Empirical Implications of the Cox, Ingersoll, Ross Theory of the Term Structure of Interest Rates", Journal of Finance 41, 617-630.

[6] Chew S.H. (1989), "An Axiomatic Utility Theory with Betweenness Property", Annals of Operations Research 19, 273-298.

[7] Chew S.H. and L. Epstein (1990), "Recursive Utility under Uncertainty", A. Khan and N. Yannelis eds, Equilibrium Theory with an Infinite Number of Commodities, Springer Verlag.

[8] Cox J., J. Ingersoll and S. Ross (1985), "A Theory of the Term Structure of Interest Rates", Econometrica 53, 385-408.

[9] Cox J.C. and S.A. Ross (1976), "The Valuation of Options for Alternative Stochastic Processes", Journal of Financial Economics 3, 145-166.

[10] Davis M. (1997), "Option pricing in incomplete markets" in M. Dempster, S. Pliska (eds): Mathematics of Derivative Securities, 216-226. Cambridge University Press.

[11] Dekel, E. (1986), "An Axiomatic Characterization of Preferences under Uncertainty", Journal of Economic Theory 40, 302-318.

[12] Doetsch G. (1970), Laplace Transformations. Springer-Verlag.

[13] Duffie D. (1988), Security Markets: Stochastic Models. Boston: Academic Press.

[14] Duffie D. and L. Epstein (1992a), "Stochastic Differential Utility", Econometrica 60, 353-394.

[15] Duffie D. and L. Epstein (1992b), "Asset Pricing with Stochastic Differential Utility", The Review of Financial Studies 5, 411-436.

[16] Duffie D. and C. Skiadas (1994), "Continuous-Time Security Pricing: A Utility Gradient Approach", Journal of Mathematical Economics 23, 107131.

[17] Epstein, L. (1992), "Behavior Under Risk: Recent Developments in Theory and Applications" in Advances in Economic Theory - Sixth World Congress, J.J. Laffont eds. Cambridge University Press. 
[18] Epstein L. and S. Zin (1989). "Substitution, Risk Aversion and the Temporal Behavior of Consumption and Asset Returns: A Theoretical Framework", Econometrica 5\%, 937-969.

[19] Föllmer H. and D. Sondermann (1986), "Hedging of contingent claims under incomplete information" in W. Hildenbrand and A. Mas-Colell (eds): Contributions to Mathematical Economics, 205-223. Amsterdam: North Holland.

[20] Föllmer H. and M. Schweizer (1991), "Hedging of contingent claims under incomplete information" in M. Davis, R. Elliott (eds): Applied Stochastic Analysis, 389-414. Gordon and Breach: London.

[21] Gerber H.U. and E.S.W. Shiu (1994), "Option pricing by Esscher transforms" (with discussions). Transactions of Society of Actuaries 46, 99-191.

[22] Gihman I.I. and A.V. Skorohod (1972), Stochastic Differential Equations. Springer-Verlag.

[23] Harrison J. and D. Kreps (1979), "Martingales and arbitrage in multiperiod securities markets", Journal of Economic Theory 20,381-408.

[24] Jarrow R. and E.R. Rosenfeld (1984), "Jump Risks and the Intertemporal Capital Asset Pricing Model", Journal of Business 57, 337-351.

[25] Karatzas I. and S. Shreve (1988), Brownian Motion and Stochastic Calculus. Springer-Verlag.

[26] Koopmans T. (1960), "Stationary Ordinal Utility and Impatience", Econometrica 28, 287-309.

[27] Kreps D. and E. Porteus (1978), "Temporal Resolution of Uncertainty and Dynamic Choice Theory", Econometrica 46, 185-200.

[28] Krylov N.V. (1980), Controlled Diffusion Processes. Springer-Verlag, Berlin.

[29] Lucas R.E. (1978), "Asset Prices in an Exchange Economy", Econometrica $46,1426-1445$.

[30] Ma C. (1992), "Valuation of Derivative Securities with Mixed PoissonBrownian Information and Recursive Utility", Ph.D. Dissertation, Chapter I, University of Toronto.

[31] Ma C. (1998), "A Discrete-Time Intertemporal Asset Pricing Model: GE Approach With Recursive Utility", Mathematical Finance 8, 249-275

[32] Ma C. (2000), "An Existence Theorem of Intertemporal Recursive Utility in the Presence of Lévy Jumps", Journal of Mathematical Economics 34, 509-526. 
[33] Ma C. (2002), "Term structure of interest rates in the presence of Lévy jumps: The HJM approach", Annals of Economics and Finance 4, 401426.

[34] Ma C. (2005), "Asset Pricing and Observational Equivalence in the Presence of Lévy Jumps" in Changing Models, Guido A. Rossi ed., Levrotto and Bella 117-149.

[35] Ma C. (2006), "Intertemporal Recursive Utility and an Equilibrium Asset Pricing Model in the Presence of Lévy Jumps", Journal of Mathematical Economics 42, 131-160.

[36] Ma C. and K. Vetzal (1995), "Pricing Options on the Market Portfolio with Discontinuous Returns under Recursive Utility", University of Waterloo, mimeo.

[37] Merton R.C. (1973), "An Intertemporal Capital Asset Pricing Model", Econometrica 41, 867-888.

[38] Merton R.C. (1976), "Option Pricing When Underlying Stock Returns Are Discontinuous", Journal of Financial Economics 3, 125-144.

[39] Naik E. and M. Lee (1990), "General Equilibrium Pricing of Options on the Market Portfolio with Discontinuous Returns", Review of Financial Studies 3, 493-521.

[40] Press, S.J. (1967), "A Compound Events Model for Security Prices", Journal of Business 40, 317-335.

[41] Protter P. (1990), Stochastic Integration and Differential Equations: A New Approach. Springer-Verlag.

[42] von Neumann J. and O. Morgenstern (1947), Theory of Games and Economic Behavior (2nd edition). Princeton University Press. 